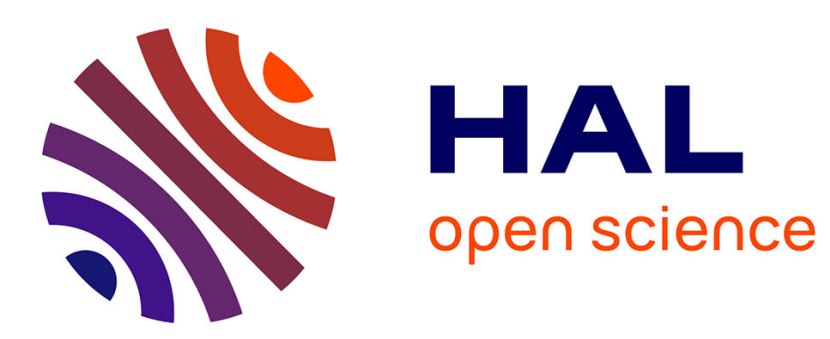

\title{
Propagation of Gibbsianness for infinite-dimensional gradient Brownian diffusions
}

\author{
David Dereudre, Sylvie Roelly
}

\section{To cite this version:}

David Dereudre, Sylvie Roelly. Propagation of Gibbsianness for infinite-dimensional gradient Brownian diffusions. 2004. hal-00003585

\section{HAL Id: hal-00003585 \\ https://hal.science/hal-00003585}

Preprint submitted on 15 Dec 2004

HAL is a multi-disciplinary open access archive for the deposit and dissemination of scientific research documents, whether they are published or not. The documents may come from teaching and research institutions in France or abroad, or from public or private research centers.
L'archive ouverte pluridisciplinaire HAL, est destinée au dépôt et à la diffusion de documents scientifiques de niveau recherche, publiés ou non, émanant des établissements d'enseignement et de recherche français ou étrangers, des laboratoires publics ou privés. 


\title{
Propagation of Gibbsianness for infinite-dimensional gradient Brownian diffusions.
}

\author{
David DeREudre* Sylvie ReLly**
}

\begin{abstract}
We study the (strong-)Gibbsian character on $\mathbb{R}^{\mathbb{Z}^{d}}$ of the law at time $t$ of an infinitedimensional gradient Brownian diffusion, when the initial distribution is Gibbsian.
\end{abstract}

AMS Classifications: 60G15 - 60G60 - 60H10 - 60J60.

KEY-WORDS: infinite-dimensional Brownian diffusion, Gibbs measure, cluster expansion.

* Université de Valenciennes et du Hainaut-Cambrésis, Le Mont Houy, 59313 Valenciennes Cedex 09, France

** Institut für Mathematik der Universität Potsdam, Postfach 601553, 14415 Potsdam, Germany; e-mail : roelly@math.uni-potsdam.de ; phone number : (49) 3319771478 ; fax number : (49) 3319771001 On leave of absence Centre de Mathématiques Appliquées, UMR C.N.R.S. 7641, École Polytechnique, 91128 Palaiseau Cedex, France. 


\section{Introduction}

Let $I$ be a finite index set and $X=\left(X_{i}(t), i \in I, t \in[0, T]\right)$ be an $\mathbb{R}^{I}$-valued diffusion which is the solution of the following finite-dimensional stochastic differential equation (s.d.e.)

$$
d X_{i}(t)=d B_{i}(t)-\frac{1}{2} \nabla_{i} h(X(t)) d t, \quad i \in I, t \in[0, T]
$$

where $h$ is a smooth function from $\mathbb{R}^{I}$ into $\mathbb{R}$ and $\left(B_{i}\right)_{i \in I}$ is a sequence of independent, real-valued Brownian motions. This stochastic dynamics corresponds to a perturbation by gradient interactions in the form of drift terms of a sequence of finitely many Brownian free dynamics.

The stationary measures for (1) are proportional to the measure

$$
\mu(d x)=\exp (-h(x)) \otimes_{i \in I} d x_{i} .
$$

It is well known that, for $h$ sufficiently regular, if the initial distribution $\nu$ of the system (1) is not the stationary one but absolutely continuous with respect to Lebesgue measure, this property propagates, that is : at each time $t>0$ the law $\nu^{t}$ of $X(t) \in \mathbb{R}^{I}$ remains absolutely continuous (with density given by $\exp -h^{t}$ for some function $h^{t}$ ).

When $I$ is replaced by $\mathbb{Z}^{d}$ and the dynamics (1) is generalised in a natural way (see (4)), the question whether the global absolute continuity of the initial distribution propagates is irrelevant since stationary measures like Gibbs measures are no more globally absolutely continuous with respect to the infinite product of Lebesgue measure, but only locally absolutely continuous. Now, the question which is relevant is the following :

\section{does Gibbsianness of the initial measure propagate?}

In fact, at each fixed time $t$ the law of $X(t)$ can behave badly in the sense that the sum of the interactions between the (infinitely many) components can explode. So, to obtain a positive answer to the above question, we restrict our study to two particular regimes which can be better controlled. In Section 3, we present the propagation of Gibbsianness for small times $t$, and in Section 4, we analyse the case of small interactions between the coordinates - but arbitrary times.

To our knowledge, these results are new ( but see also [18]) which are related to the propagation of Gibbsianness under a stochastic evolution like a diffusion with values in a continuous space (here the state space is the infinite-dimensional vector space $\mathbb{R}^{\mathbb{Z}^{d}}$ ). We were inspired by the nice work of van Enter, Fernandez, den Hollander and Redig, who consider in [8] the question of possible loss and recovery of Gibbsianness in the context of Interacting Particle Systems with values in $\{-1,+1\}^{\mathbb{Z}^{d}}$ which follow a high-temperature Glauber dynamics. They treat several cases and can exhibit situations where the process at time $t$ is strong Gibbsian (in a sense to be defined below), and other situations where it is not. See also [19] for related results for Kawasaki dynamics. Unfortunately, since our state space $\mathbb{R}^{\mathbb{Z}^{d}}$ is unbounded, we cannot use all the criteria they have at their disposition (in particular, the criterion of non-Gibbsianness contained in [11]) to test the Gibbsianness/non-Gibbsianness of $\nu^{t}$. So our present results only concern situations for which the Gibbsianness is conserved. We hope to extend them soon to some non-Gibbsian example. 


\section{Gibbs measures and infinite-dimensional gradient diffu- sions : the framework of our study}

Let us first introduce some definitions and notations.

An interaction potential - or interaction - $\phi$ on $\mathbb{R}^{\mathbb{Z}^{d}}$ is a collection of functions $\phi_{\Lambda}$ from $\mathbb{R}^{\mathbb{Z}^{d}}$ into $\mathbb{R} \cup\{+\infty\}$ where $\Lambda$ varies in the set of finite subsets of $\mathbb{Z}^{d}$. Each $\phi_{\Lambda}$ is supposed to be measurable with respect to $\mathcal{F}_{\Lambda}$, the $\sigma$-algebra generated by the canonical projection on $\mathbb{R}^{\Lambda}$; that is for any $x \in \mathbb{R}^{\mathbb{Z}^{d}}$,

$$
\phi_{\Lambda}(x)=\phi_{\Lambda}\left(x_{\Lambda}\right)
$$

where $x_{\Lambda}$ is the projection on $\mathbb{R}^{\Lambda}$ of $x$.

The interaction $\phi$ is said to be of finite range if it satisfies :

(FR) $\quad \exists r>0$, diameter $\Lambda>r \Longrightarrow \phi_{\Lambda} \equiv 0$

The interaction $\phi$ is said to be regular bounded if it satisfies :

(RB) $\forall \Lambda, \phi_{\Lambda}$ is $\mathcal{C}^{3}$, bounded with bounded derivatives.

The interaction $\phi$ is said to be absolutely summable if it satisfies :

$$
\forall i \in \mathbb{Z}^{d}, \sum_{\Lambda \ni i}\left\|\phi_{\Lambda}\right\|_{\infty}=\sum_{\Lambda \ni i} \sup _{x \in \mathbb{R}^{\mathbb{Z}^{d}}}\left|\phi_{\Lambda}(x)\right|<+\infty
$$

When an interaction $\phi$ is (AS) one can define the collection $h^{\phi}=\left(h_{\Lambda}^{\phi}\right)_{\Lambda \subset \mathbb{Z}^{d}}$ of associated Hamiltonian functions on $\mathbb{R}^{\mathbb{Z}^{d}}$ by

$$
h_{\Lambda}^{\phi}=\sum_{\Lambda^{\prime}: \Lambda^{\prime} \cap \Lambda \neq \emptyset} \phi_{\Lambda^{\prime}}
$$

More generally, we note for $x, y \in \mathbb{R}^{\mathbb{Z}^{d}}$ and $\Lambda, \Delta \subset \mathbb{Z}^{d}$

$$
h_{\Lambda, \Delta}^{\phi}(x, y)=\sum_{\substack{\Lambda^{\prime}: \Lambda^{\prime} \cap \Lambda \neq \emptyset \\ \Lambda^{\prime} \subset \Lambda \cup \Delta}} \phi_{\Lambda^{\prime}}\left(x_{\Lambda} y_{\Delta \backslash \Lambda}\right),
$$

where $x_{\Lambda} y_{\Delta \backslash \Lambda}$ is the element in $\mathbb{R}^{\mathbb{Z}^{d}}$ equal to $x$ on $\Lambda, y$ on $\Delta \backslash \Lambda$ and 0 outside of $\Lambda \cup \Delta$. For example, $h_{\Lambda, \mathbb{Z}^{d}}^{\phi}(x, x)$ coincides with $h_{\Lambda}^{\phi}(x)$. Furthermore, $h_{\Lambda, \emptyset}^{\phi}(x)=\sum_{\Lambda^{\prime} \subset \Lambda} \phi_{\Lambda^{\prime}}\left(x_{\Lambda^{\prime}}\right)$ is a function of $x_{\Lambda}$. To recall this property, we will sometimes denote it by $h_{\Lambda, \emptyset}^{\phi}\left(x_{\Lambda}\right)$.

In fact, as soon as the series on the right-hand side of (2) converges pointwise, one can define a Hamiltonian function associated to a (possibly non absolutely) summable interaction. To simplify we will always denote by $h_{i}^{\phi}$ the function $h_{\{i\}}^{\phi}\left(i \in \mathbb{Z}^{d}\right)$, by $h_{\Lambda, \Delta}^{\phi}(x)$ the function $h_{\Lambda, \Delta}^{\phi}(x, x)\left(\Lambda, \Delta \in \mathbb{Z}^{d}, x \in \mathbb{R}^{\mathbb{Z}^{d}}\right)$.

We call $\rho$ a Gibbsian measure on $\mathbb{R}^{\mathbb{Z}^{d}}$ associated to the reference measure $m$ and to an interaction $\phi$ for which the series (2) converges if it satisfies the system of DobrushinLanford-Ruelle (DLR) equations :

$$
\rho\left(d x_{i} / x_{j}, j \neq i\right)=\frac{1}{z_{i}} \exp -\left(h_{i}^{\phi}(x)\right) m\left(d x_{i}\right), i \in \mathbb{Z}^{d} .
$$


The set of such measures will be denoted by $\mathcal{G}(\phi, m)$. (For general references on Gibbs measures, see [13] and [24].)

The measure $\rho$ will be called strong Gibbsian if the associated interaction is absolutely summable, i.e. satisfies (AS).

Let $\varphi$ be a so-called dynamical interaction on $\mathbb{R}^{\mathbb{Z}^{d}}$, having a $\mathcal{C}^{2}$-regularity and satisfying (FR). The associated hamilton function $h_{i}^{\varphi}$, denoted by $h_{i}$ to simplify, is also $\mathcal{C}^{2}$. We can now consider the following infinite-dimensional system given by :

$$
\left\{\begin{array}{l}
d X_{i}(t)=d B_{i}(t)-\frac{1}{2} \nabla_{i} h_{i}(X(t)) d t, i \in \mathbb{Z}^{d}, t \in[0, T] \\
X(0) \simeq \nu
\end{array}\right.
$$

where $\nu$ is a probability measure on $\mathbb{R}^{\mathbb{Z}^{d}}$. We will precise in Section 3 (resp. Section 4 ) the exact assumptions on $h$ and $\nu$ we take to assure that the infinite-dimensional stochastic system (3) has a unique strong Markovian solution $X$ with values in the infinite product of continuous trajectories $\Omega_{T}=\mathcal{C}([0, T], \mathbb{R})^{\mathbb{Z}^{d}}$. Deuschel described in $([5],[6])$ the Gibbsian structure on the path space $\Omega_{T}$ of the law $Q^{\nu}$, when the initial distribution $\nu$ itself is Gibbsian (associated to an initial interaction $\tilde{\varphi}$ ). Later, this result was completed and generalised in [2], by showing a bijection between the set of Gibbs measures associated to the initial interaction $\tilde{\varphi}$ on $\mathbb{R}^{\mathbb{Z}^{d}}$ and a set of Gibbs measures on the path space $\Omega_{T}$ describing the full dynamics. Having a Gibbs representation of $Q^{\nu}$ on the path level (even a strong Gibbsian one, see [4] Corollary 2), we would like to know if at each time $t$, the law $\nu^{t}$ of $X(t)$, a probability measure on $\mathbb{R}^{\mathbb{Z}^{d}}$, remains strong Gibbsian. Clearly, $\nu^{t}$ is the projection at time $t$ of $Q^{\nu}$, but projections are maps which do not conserve a priori the Gibbsianness (see the famous examples of [9], and also [10], [11] amoung others). In [2], we remarked that, projecting at time 0 a general strong Gibbs measure on the path space, the image measure which is obtained on the state space preserves a Gibbsian form in the following weak sense : it is associated to a modification (cf. [13] Section 1.3, for the exact definition), roughly speaking to a family of compatible local densities with respect to a reference measure. But the regularity of the density and the existence of an underlying nice interaction potential is completely unclear. In the Remarks after Proposition 2.5 in [2], we referred the reader to the work of Kozlov to clarify this question. This is the object of this paper, not only for the projection at time 0 but also at time $t>0$.

The challenge is to control the evolution of an initial absolutely summable interaction $\tilde{\varphi}$ under the dynamics (3). It is clear that, even if $\tilde{\varphi}$ is of finite range this property immediately disappears for any time $t>0$ since the Brownian motions carry instantaneously information between all the coordinates. So, to assure that at time $t$, the process is still Gibbsian and associated to a "good" interaction, i.e. an absolutely summable one, we are obliged to restrict our study to two cases; first for small times $t$, which implies that the process stays close to the initial Gibbsian condition. Secondly, for small dynamical interaction $\varphi$ between the coordinates, which assures that the sum of the initial interaction and the interaction induced by the dynamics does not explode. 


\section{Propagation of Gibbsianness during a short stochastic diffusive evolution}

Let us consider the infinite-dimensional gradient system (3) introduced in Section 2 where the initial distribution is Gibbsian. We have the following result.

Theorem 1 Let $Q^{\nu}$ be the law on $\Omega=\mathcal{C}\left(\mathbb{R}^{+}, \mathbb{R}\right)^{\mathbb{Z}^{d}}$ of the infinite-dimensional diffusion solution of

$$
\left\{\begin{array}{l}
d X_{i}(t)=d B_{i}(t)-\frac{1}{2} \nabla_{i} h_{i}(X(t)) d t, i \in \mathbb{Z}^{d}, t>0 \\
X(0) \simeq \nu
\end{array}\right.
$$

where $\nu \in \mathcal{G}(\tilde{\varphi}, d x)$ with support included in $l^{1}(\gamma)$, with $\gamma=\left(e^{-\alpha|i|}\right)_{i \in \mathbb{Z}^{d}}, \alpha>0$. Let us moreover suppose that

- the initial interaction $\tilde{\varphi}$ is of finite range (FR) and each $\tilde{\varphi}_{\Lambda}$ is Lipschitz continuous (uniformly in $\Lambda$ )

- the dynamical interaction $\varphi$ is of finite range (FR), and each $\varphi_{\Lambda}$ is $\mathcal{C}^{2}$ with bounded derivatives of order 1 and 2 (uniformly in $\Lambda$ ).

Then, there exists a time $t_{0}>0$ depending only on $\tilde{\varphi}$ and $\varphi$ such that, for any $t \leq t_{0}$,

$$
\left\{\nu^{t}=Q^{\nu} \circ X(t)^{-1}: \nu \in \mathcal{G}(\tilde{\varphi}, d x)\right\} \subset \mathcal{G}\left(\varphi^{t}, d x\right)
$$

where $\varphi^{t}$ is an absolutely summable $(A S)$ interaction depending only on the initial and dynamical interactions $\tilde{\varphi}$ and $\varphi$.

Remark 1 One can make explicit some additional assumptions on $\tilde{\varphi}$ in order to assure that $\mathcal{G}(\tilde{\varphi}, d x)$ contains at least one measure with support included in $l^{1}(\gamma)$. For example suppose there exists $a>0, b \geq 0$ such that for each $i \in \mathbb{Z}^{d}$

$$
\begin{aligned}
& \text { (i) } \forall x \in \mathbb{R}, \quad x \nabla_{i} \tilde{\varphi}_{i}(x) \geq a|x|-b \\
& \text { (ii) } a>\sum_{\substack{\Lambda \ni i \\
\# \Lambda>1}}\left\|\nabla_{i} \tilde{\varphi}_{\Lambda}\right\|_{\infty} .
\end{aligned}
$$

Then there exists $\nu \in \mathcal{G}(\tilde{\varphi}, d x)$ satisfying $\int\|x\|_{\gamma} \nu(d x)<+\infty$ where $\|x\|_{\gamma}=: \sum_{i \in \mathbb{Z}^{d}}\left|x_{i}\right| e^{-\alpha|i|}$. This obviously implies that $\nu\left\{x:\|x\|_{\gamma}<+\infty\right\}=1$.

The Proof of Remark 1 is postponed to the end of the section.

\section{Proof of Theorem 1 :}

The proof is based on an approximation of $\nu^{t}$ by a sequence of probability measures $\nu_{\Lambda}^{t}$, which are the laws at time $t$ of finite-dimensional systems. It will be relatively easy to obtain a Gibbs representation for each $\nu_{\Lambda}^{t}$. But the delicate point will be the convergence of their associated Hamiltonian functions towards a limiting function, which will be a good candidate as Hamiltonian function associated to $\nu^{t}$.

Let us first recall a representation theorem, which we will use for the initial Gibbs measure $\nu$ (Theorems 7.12 and 7.26 in [13]). 
Lemma 1 The probability measure $\nu$, like every element of $\mathcal{G}(\tilde{\varphi}, d x)$, is a mixture of elements of ex $\mathcal{G}(\tilde{\varphi}, d x)$, where ex $\mathcal{G}(\tilde{\varphi}, d x)$ is the set of extremal Gibbs measures $\mu$ which are characterized by the following property : there exists $y \in \mathbb{R}^{\mathbb{Z}^{d}}$ such that

$$
\mu=\lim _{\Lambda \nearrow \mathbb{Z}^{d}} \mu_{\Lambda, y} \otimes \delta_{y_{\Lambda^{c}}} \text { where } \mu_{\Lambda, y}\left(d x_{\Lambda}\right)=\frac{1}{\tilde{Z}_{\Lambda, y}} e^{-\tilde{h}_{\Lambda, \Lambda^{c}}(x, y)} d x_{\Lambda} .
$$

The family of $\mu_{\Lambda, y}$ is in fact the family of finite volume specifications with fixed boundary condition $y$.

The limit in the above Lemma is taken in the following sense: for any increasing sequence $\Lambda_{n}$ of finite subsets in $\mathbb{Z}^{d}$ converging to $\mathbb{Z}^{d}$ when $n$ goes to infinity, $\mu_{\Lambda_{n}, y} \otimes \delta_{y_{\Lambda_{n}^{c}}}$ converges in the local convergence topology towards $\mu$.

We first prove the theorem in the case where $\nu \in \operatorname{ex} \mathcal{G}(\tilde{\varphi}, d x)$.

Let $\left(\nu_{\Lambda, y}\right)_{\Lambda \subset \mathbb{Z}^{d}}$ be the approximating sequence of $\nu$ defined by (5). For $\Lambda \subset \mathbb{Z}^{d}$ fixed and any $i \in \Lambda$, we introduce the $i$-decoupled infinite measure $\nu_{\Lambda, y}^{i}$ as follows :

$$
\nu_{\Lambda, y}^{i}\left(d x_{\Lambda}\right)=\frac{1}{\tilde{Z}_{\Lambda, y}} e^{-\tilde{h}_{\Lambda \backslash i, \Lambda^{c}}(x, y)} d x_{\Lambda \backslash i} d x_{i} .
$$

Since $\tilde{h}_{\Lambda, \Lambda^{c}}(x, y)=\tilde{h}_{\Lambda \backslash i, \Lambda^{c}}(x, y)+\tilde{h}_{i}\left(x_{\Lambda} y_{\Lambda^{c}}\right)$, we obtain

$$
\nu_{\Lambda, y}^{i}\left(d x_{\Lambda}\right)=e^{\tilde{h}_{i}\left(x_{\Lambda} y_{\Lambda^{c}}\right)} \nu_{\Lambda, y}\left(d x_{\Lambda}\right) .
$$

Let us remark that $\nu_{\Lambda, y}^{i} \otimes \delta_{y_{\Lambda^{c}}}$ converges in $\Lambda$ towards a measure $\nu^{i}$ on $\mathbb{R}^{\mathbb{Z}^{d}}$ which is absolutely continuous with respect to $\nu$ and satisfies :

$$
\nu^{i}(d x)=e^{\tilde{h}_{i}(x)} \nu(d x) .
$$

In the same way, we denote by $\mu_{\Lambda}$ and $\mu_{\Lambda}^{i}$ the following measures (not necessary finite) :

$$
\mu_{\Lambda}\left(d x_{\Lambda}\right)=e^{-h_{\Lambda, \emptyset}\left(x_{\Lambda}\right)} d x_{\Lambda}, \quad \mu_{\Lambda}^{i}\left(d x_{\Lambda}\right)=e^{-h_{\Lambda \backslash i, \emptyset}\left(x_{\Lambda \backslash i}\right)} d x_{\Lambda \backslash i} d x_{i} .
$$

Then

$$
\mu_{\Lambda}^{i}\left(d x_{\Lambda}\right)=e^{h_{i, \Lambda}\left(x_{\Lambda}\right)} \mu_{\Lambda}\left(d x_{\Lambda}\right) .
$$

Let us now introduce the following finite-dimensional approximation of the dynamics (4) :

$$
d X_{i}(t)=d B_{i}(t)-\frac{1}{2} \nabla_{i} h_{i, \Lambda}\left(X_{\Lambda}(t)\right) d t, \quad \forall i \in \Lambda, t>0 .
$$

Remark that we also could write the drift as $-\frac{1}{2} \nabla_{i} h_{\Lambda, \emptyset}\left(X_{\Lambda}(t)\right)$. Under this form, $\mu_{\Lambda}$ is clearly a reversible measure associated to this dynamics.

We denote by $Q_{\Lambda}^{x_{\Lambda}}$ the law on $\mathcal{C}\left(\mathbb{R}^{+}, \mathbb{R}\right)^{\Lambda}$ of the solution of (9) when the initial condition is $x_{\Lambda} \in \mathbb{R}^{\Lambda}$.

We now introduce, in the same way as above, some decoupled (infinite- and finite-dimensional) dynamics at the site $i$ :

$$
\left\{\begin{array}{l}
d X_{j}(t)=d B_{j}(t)-\frac{1}{2} \nabla_{j} h_{j, \mathbb{Z}^{d} \backslash i}(X(t)) d t, \quad \forall j \in \mathbb{Z}^{d} \backslash\{i\}, t>0 \\
d X_{i}(t)=d B_{i}(t), \quad t>0 .
\end{array}\right.
$$


$Q^{x, i}$ denotes the law of the solution of (10) with deterministic initial condition $x \in \mathbb{R}^{\mathbb{Z}^{d}}$. These dynamics are useful since they are simpler than the undecoupled ones, and we will prove that the law $\nu^{t}$ of the gradient system at time $t$ is absolutely continuous with respect to the law at time $t$ of the above decoupled system.

We also consider the finite-dimensional approximation of (10) :

$$
\left\{\begin{array}{l}
d X_{j}(t)=d B_{j}(t)-\frac{1}{2} \nabla_{j} h_{j, \Lambda \backslash i}\left(X_{\Lambda \backslash i}(t)\right) d t, \quad \forall j \in \Lambda \backslash i, t>0 \\
d X_{i}(t)=d B_{i}(t) .
\end{array}\right.
$$

We denote by $Q_{\Lambda}^{x_{\Lambda}, i}$ the law on $\mathcal{C}\left(\mathbb{R}^{+}, \mathbb{R}\right)^{\Lambda}$ of the solution of (11) when the initial condition is $x_{\Lambda} \in \mathbb{R}^{\Lambda} . \mu_{\Lambda}^{i}$ is a reversible measure associated to this dynamics.

Since the solution of (4) (when it exists) is Markovian, one has : $Q^{\nu}=\int Q^{x} \nu(d x)$. More generally, for any measure $\mu$, we denote by $Q^{\mu}$ (resp. $Q^{\mu, i}, Q_{\Lambda}^{\mu}$ or $Q_{\Lambda}^{\mu, i}$ ) the mixture of $Q^{x}$ under $\mu: Q^{\mu}=\int Q^{x} \mu(d x)\left(\operatorname{resp} . \int Q^{x, i} \mu(d x), \int Q_{\Lambda}^{x} \mu(d x)\right.$ or $\left.\int Q_{\Lambda}^{x, i} \mu(d x)\right)$.

We also define the projections at time $t$ of these measures :

$\nu^{t}=Q^{\nu} \circ X(t)^{-1}, \nu^{t, i}=Q^{\nu^{i}, i} \circ X(t)^{-1}, \nu_{\Lambda, y}^{t}=Q_{\Lambda}^{\nu_{\Lambda, y}} \circ X(t)^{-1}, \nu_{\Lambda, y}^{t, i}=Q_{\Lambda}^{\nu_{\Lambda, y}^{i}, i} \circ X(t)^{-1}$.

Lemma 2 For each $t \in[0, T]$ and $i \in \mathbb{Z}^{d}$, the following weak convergences hold:

$$
\lim _{\Lambda \nearrow \mathbb{Z}^{d}} \nu_{\Lambda, y}^{t}=\nu^{t} \text { and } \quad \lim _{\Lambda \nearrow \mathbb{Z}^{d}} \nu_{\Lambda, y}^{t, i}=\nu^{t, i}
$$

\section{Proof :}

We only prove the first convergence. The proof of the second one is analogous.

Under the assumptions satisfied by $\varphi$ in Theorem 1, it is simple to verify that for any initial deterministic condition $x \in l^{1}(\gamma)=\left\{y=\left(y_{i}\right)_{i \in \mathbb{Z}^{d}} \in \mathbb{R}^{\mathbb{Z}^{d}}:\|y\|_{\gamma}<+\infty\right\}$, a strong solution of $(4)$ exists in $\mathcal{C}\left(\mathbb{R}^{+}, l^{1}(\gamma)\right)$. It is obtained as limit of finite dimensional diffusions solution of (9). More precisely, let $\Lambda_{n}$ be an increasing sequence of finite subsets in $\mathbb{Z}^{d}$ converging to $\mathbb{Z}^{d}$ when $n$ goes to infinity. To clarify the notations, instead of using the canonical processes, for $x \in \mathbb{R}^{\mathbb{Z}^{d}}$ we denote by $X^{x}$ the solution of (4) with $\nu=\delta_{x}$ and by $X^{(n), x}$ the (infinite-dimensional) process with initial condition $x$ whose restriction on $\Lambda_{n}$ solves (9) with $\Lambda=\Lambda_{n}$ and whose coordinates outside $\Lambda_{n}$ are frozen in $x_{\Lambda_{n}^{c}}$. So the law of $X^{x}$ is equal to $Q^{x}$ and the law of $X^{(n), x}$ is equal to $Q_{\Lambda_{n}}^{x_{\Lambda_{n}}} \otimes \delta_{x_{\Lambda_{n}^{c}}}$. Following analogous techniques as the one used in [27] Theorem 4.1 (or [7], [26] if the interaction is reduced to a pair interaction), we now prove that, for any $T>0, X^{(n), x}$ is a Cauchy sequence in $L^{1}\left(\mathcal{C}\left([0, T], l^{1}(\gamma)\right)\right)$.

Let $r$ the range of $\varphi$ and $k>0$ the supremum of a Lipschitz constant for $\nabla_{j} h_{j}$ (uniform in $j$ ) and a bound for $\sup _{x}\left|\nabla_{j} h_{j}(x)\right|$. Let $m<n$ and let $\Lambda_{m}^{\circ}$ denote the $r$-interior of $\Lambda_{m}$ defined by $\Lambda_{m}^{\circ}=\left\{j \in \Lambda_{m}: \forall k\right.$ with $\left.|k-j| \leq r, k \in \Lambda_{m}\right\}$. So $\Lambda_{m}^{\circ} \subset \Lambda_{m} \subset \Lambda_{n}$.

For $i \in \Lambda_{m}^{\circ}$,

$$
\begin{aligned}
\left|X_{i}^{(n), x}(t)-X_{i}^{(m), x}(t)\right| & =\frac{1}{2}\left|\int_{0}^{t} \nabla_{i} h_{i, \Lambda_{n}}\left(X^{(n), x}(s)\right)-\nabla_{i} h_{i, \Lambda_{m}}\left(X^{(m), x}(s)\right) d s\right| \\
& \leq \frac{k}{2} \sum_{\{j:|j-i| \leq r\}} \int_{0}^{t}\left|X_{j}^{(n), x}(s)-X_{j}^{(m), x}(s)\right| d s
\end{aligned}
$$


Thus,

$$
E\left(\sup _{s \leq t}\left|X_{i}^{(n), x}(s)-X_{i}^{(m), x}(s)\right|\right) \leq \frac{k}{2} \sum_{\{j:|j-i| \leq r\}} \int_{0}^{t} E\left(\sup _{u \leq s}\left|X_{j}^{(n), x}(u)-X_{j}^{(m), x}(u)\right|\right) d s .
$$

For $i \in \Lambda_{m} \backslash \Lambda_{m}^{\circ}$

$$
\begin{aligned}
& \left|X_{i}^{(n), x}(t)-X_{i}^{(m), x}(t)\right| \\
= & \frac{1}{2}\left|\int_{0}^{t} \nabla_{i} h_{i, \Lambda_{n}}\left(X^{(n), x}(s)\right)-\nabla_{i} h_{i, \Lambda_{m}}\left(X^{(m), x}(s)\right) d s\right| \\
\leq & \frac{k}{2} \sum_{\{j:|j-i| \leq r\} \cap \Lambda_{m}} \int_{0}^{t}\left|X_{j}^{(n), x}(s)-X_{j}^{(m), x}(s)\right| d s+\frac{1}{2}\left|\int_{0}^{t} \sum_{\substack{\Lambda^{\prime}: \Lambda^{\prime} \not \subset \Lambda_{m} \\
\Lambda^{\prime} \subset \Lambda_{n}}} \nabla_{i} \varphi_{\Lambda^{\prime}}\left(X^{(m), x}(s)\right) d s\right|
\end{aligned}
$$

Thus,

$$
E\left(\sup _{s \leq t}\left|X_{i}^{(n), x}(s)-X_{i}^{(m), x}(s)\right|\right) \leq \frac{k}{2} \sum_{\{j:|j-i| \leq r\}} \int_{0}^{t} E\left(\sup _{r \leq s}\left|X_{j}^{(n), x}(r)-X_{j}^{(m), x}(r)\right|\right) d s+\frac{k}{2} t .
$$

For $i \in \Lambda_{n} \backslash \Lambda_{m}$, we have

$$
\left|X_{i}^{(n), x}(t)-X_{i}^{(m), x}(t)\right|=\left|B_{i}(t)-\frac{1}{2} \int_{0}^{t} \nabla_{i} h_{i, \Lambda_{n}}\left(X^{(n), x}(s)\right) d s\right| ;
$$

thus, using Doob inequality for $B_{i}$,

$$
E\left(\sup _{s \leq t}\left|X_{i}^{(n), x}(s)-X_{i}^{(m), x}(s)\right|\right) \leq 2 \sqrt{t}+\frac{k}{2} t .
$$

For $i \in \Lambda_{n}^{c},\left|X_{i}^{(n), x}(t)-X_{i}^{(m), x}(t)\right| \equiv 0$.

To obtain the desired Cauchy property, we apply an infinite-dimensional version of Gronwall's Lemma. It is simple to verify (see for example [25] Lemme1, page 197) that, if C is a positive constant which satisfies : for any $j \in \mathbb{Z}^{d}, \frac{k}{2} \sum_{\{i:|j-i| \leq r\}} \gamma_{i} \leq C \gamma_{j}$, (one can take $\left.C=e^{\alpha r} \#\left\{k \in \mathbb{Z}^{d},|k| \leq r\right\}\right)$, then,

$$
\begin{aligned}
E\left(\sup _{s \leq t}\left\|X^{(n), x}(s)-X^{(m), x}(s)\right\|_{\gamma}\right) & =E\left(\sup _{s \leq t} \sum_{i \in \mathbb{Z}^{d}} \gamma_{i}\left|X_{i}^{(n), x}(s)-X_{i}^{(m), x}(s)\right|\right) \\
& \leq\left(2 \sqrt{t}+\frac{k}{2} t\right)\left(\sum_{i \in \Lambda_{n} \backslash \Lambda_{m}^{\circ}} \gamma_{i}\right) \exp \frac{k C}{2} t .
\end{aligned}
$$

Since $\gamma \in l^{1}\left(\mathbb{Z}^{d}\right)$ the right hand side of this inequality goes to 0 when $n$ and $m$ are large enough - uniformly in $x$ - we conclude that $X^{(n), x}$ converges in $L^{1}\left(\mathcal{C}\left([0, T], l^{1}(\gamma)\right)\right)$ uniformly in $x$ towards $X^{x}$.

Moreover, with similar computations as above, one obtains the following Lipschitz regularity of $X^{x}$ as a function of $x$ :

$$
\exists C^{\prime}>0, E\left(\sup _{s \leq t}\left\|X^{x}(s)-X^{y}(s)\right\|_{\gamma}\right) \leq\|x-y\|_{\gamma} e^{C^{\prime} t}
$$


Now, the law of the solution of (4) is just a mixture under $\nu$ of the laws $Q^{x}$. We may claim this since the support of $\nu$ is included in $l^{1}(\gamma)$.

Let us now prove the local convergence of $\nu_{\Lambda_{n}, y}^{t}$ towards $\nu^{t}$. Let $g$ be a $\Delta$-local Lipschitz function on $\mathbb{R}^{\mathbb{Z}^{d}}$ with Lipschitz constant $K_{g}$ and fix $m<n$ large enough to have $\Delta \subset \Lambda_{m}(\subset$ $\left.\Lambda_{n}\right)$. We have

$$
\begin{aligned}
& \left|\int g\left(x_{\Delta}\right) \nu^{t}(d x)-\int g\left(x_{\Delta}\right) \nu_{\Lambda_{n}, y}^{t}\left(d x_{\Lambda_{n}}\right)\right| \\
& =\left|\int g\left(X_{\Delta}(t)\right) Q^{\nu}(d X)-\int g\left(X_{\Delta}(t)\right) Q_{\Lambda_{n}}^{\nu_{\Lambda_{n}, y}}\left(d X_{\Lambda_{n}}\right)\right| \\
& =\left|\int_{\mathbb{R}^{\mathbb{Z}^{d}}} \int g\left(X_{\Delta}(t)\right) Q^{x}(d X) \nu(d x)-\int_{\mathbb{R}^{\Lambda_{n}}} g\left(X_{\Delta}(t)\right) Q_{\Lambda_{n}}^{x_{\Lambda_{n}}}\left(d X_{\Lambda_{n}}\right) \nu_{\Lambda_{n}, y}\left(d x_{\Lambda_{n}}\right)\right| \\
& \leq C_{1}+C_{2}+C_{3}
\end{aligned}
$$

where

$$
\begin{aligned}
C_{1} & =\int_{\mathbb{R}^{\mathbb{Z}^{d}}}\left|\int g\left(X_{\Delta}(t)\right) Q^{x}(d X)-\int g\left(X_{\Delta}(t)\right) Q_{\Lambda_{m}}^{x_{\Lambda_{m}}}(d X)\right| \nu(d x) \\
C_{2} & =\left|\iint g\left(X_{\Delta}(t)\right) Q_{\Lambda_{m}}^{x_{\Lambda_{m}}}(d X) \nu(d x)-\iint g\left(X_{\Delta}(t)\right) Q_{\Lambda_{m}}^{x_{\Lambda_{m}}}(d X) \nu_{\Lambda_{n}, y}\left(d x_{\Lambda_{n}}\right)\right| \\
C_{3} & =\int_{\mathbb{R}^{\Lambda_{n}}}\left|\int g\left(X_{\Delta}(t)\right) Q_{\Lambda_{m}}^{x_{\Lambda_{m}}}(d X)-\int g\left(X_{\Delta}(t)\right) Q_{\Lambda_{n}}^{x_{\Lambda_{n}}}(d X)\right| \nu_{\Lambda_{n}, y}\left(d x_{\Lambda_{n}}\right) .
\end{aligned}
$$

With the above notations,

$$
\begin{aligned}
C_{1} & =\int_{\mathbb{R}^{\mathbb{Z}^{d}}} E\left|g\left(X_{\Delta}^{x}(t)\right)-g\left(X_{\Delta}^{(m), x}(t)\right)\right| \nu(d x) \\
& \leq K_{g} \int_{\mathbb{R}^{\mathbb{Z}^{d}}} E\left|X_{\Delta}^{x}(t)-X_{\Delta}^{(m), x}(t)\right| \nu(d x) \\
& \leq K_{g} \int_{\mathbb{R}^{\mathbb{Z}^{d}}} E \sum_{i \in \Delta}\left|X_{i}^{x}(t)-X_{i}^{(m), x}(t)\right| \nu(d x) \\
& \leq K_{g}\left(\inf _{i \in \Delta} \gamma_{i}\right)^{-1} \int_{\mathbb{R}^{\mathbb{Z}^{d}}} E \sum_{i \in \Delta} \gamma_{i}\left|X_{i}^{x}(t)-X_{i}^{(m), x}(t)\right| \nu(d x) \\
& \leq K_{g}\left(\inf _{i \in \Delta} \gamma_{i}\right)^{-1} \int_{\mathbb{R}^{\mathbb{Z}^{d}}} E\left(\left\|X^{x}(t)-X^{(m), x}(t)\right\|_{\gamma}\right) \nu(d x) \\
& \leq c_{1}\left(\sum_{i \in\left(\Lambda_{m}^{\circ}\right)^{c}} \gamma_{i}\right),
\end{aligned}
$$

using (12) for the last inequality, where $c_{1}>0$ is a constant depending only on $t, \varphi, g, r, \Delta, \gamma$. In the same way, one has

$$
\begin{aligned}
C_{3} & =\int_{\mathbb{R}^{\Lambda_{n}}} E\left|g\left(X_{\Delta}^{(m), x}(t)\right)-g\left(X_{\Delta}^{(n), x}(t)\right)\right| \nu_{\Lambda_{n}, y}\left(d x_{\Lambda_{n}}\right) \\
& \leq K_{g} \int_{\mathbb{R}^{\Lambda_{n}}} E\left|X_{\Delta}^{(m), x}(t)-X_{\Delta}^{(n), x}(t)\right| \nu_{\Lambda_{n}, y}\left(d x_{\Lambda_{n}}\right) \\
& \leq K_{g}\left(\inf _{i \in \Delta} \gamma_{i}\right)^{-1} \int E\left(\left\|X^{(m), x}(t)-X^{(n), x}(t)\right\|_{\gamma}\right) \nu_{\Lambda_{n}, y}\left(d x_{\Lambda_{n}}\right) \\
& \leq c_{1}\left(\sum_{i \in\left(\Lambda_{m}^{\circ}\right)^{c}} \gamma_{i}\right) .
\end{aligned}
$$


The second term is controlled in the following way :

$$
C_{2}=\left|\int E\left(g\left(X_{\Delta}^{(m), x}(t)\right)\right) \nu(d x)-\int E\left(g\left(X_{\Delta}^{(m), x}(t)\right)\right) \nu_{\Lambda_{n}, y}\left(d x_{\Lambda_{n}}\right)\right| .
$$

But, for $m$ fixed, $X_{\Delta}^{(m), x}(t)$ and then $E\left(g\left(X_{\Delta}^{(m), x}(t)\right)\right.$ are $\Lambda_{m}$-local functions in $x$ depending continuously on $x_{\Lambda_{m}}$. So, thanks the local convergence of the finite-volume specifications $\nu_{\Lambda_{n}, y}$ towards $\nu$ when $n$ goes to infinity, this term vanishes for $m$ fixed and $n$ going to infinity.

To complete the proof of the convergence of $\nu_{\Lambda_{n}, y}^{t}$ towards $\nu^{t}$ it remains now to take $m$ large enough in such a way that $\left(\sum_{i \in\left(\Lambda_{m}^{\circ}\right)^{c}} \gamma_{i}\right)$ (and thus $\left.C_{1}+C_{3}\right)$ stays as small as necessary.

To prove that $\nu^{t}$ is Gibbsian, we will use the fact that $\nu^{t}$ is absolutely continuous with respect to $\nu^{t, i}$, which itself is a consequence of the absolute continuity of $\nu_{\Lambda, y}^{t}$ with respect to $\nu_{\Lambda, y}^{t, i}$. Let us start with a nice representation of this density $\frac{d \nu_{\Lambda, y}^{t}}{d \nu_{\Lambda, y}^{t, i}}$.

Lemma 3 For each $t>0, \Lambda \subset \mathbb{Z}^{d}$ and any $i \in \Lambda$,

$$
\frac{d \nu_{\Lambda, y}^{t}}{d \nu_{\Lambda, y}^{t, i}}\left(x_{\Lambda}\right)=e^{-\tilde{h}_{i}\left(x_{\Lambda} y_{\Lambda^{c}}\right)} \frac{E_{Q_{\Lambda}^{x_{\Lambda}}}\left(e^{f_{\Lambda, y}\left(X_{\Lambda}(t)\right)-f_{\Lambda, y}\left(x_{\Lambda}\right)}\right)}{E_{Q_{\Lambda, i}^{x_{\Lambda}}}\left(e^{f_{\Lambda \backslash i, y}\left(X_{\Lambda}(t)\right)-f_{\Lambda \backslash i, y}\left(x_{\Lambda}\right)}\right)},
$$

where

$$
f_{\Lambda, y}(x)=h_{\Lambda, \emptyset}\left(x_{\Lambda}\right)-\tilde{h}_{\Lambda, \Lambda^{c}}(x, y)
$$

Proof :

We have :

$$
\frac{d \nu_{\Lambda, y}^{t}}{d \nu_{\Lambda, y}^{t, i}}\left(x_{\Lambda}\right)=\frac{d \nu_{\Lambda, y}^{t}}{d \mu_{\Lambda}}\left(x_{\Lambda}\right) \frac{d \mu_{\Lambda}}{d \mu_{\Lambda}^{i}}\left(x_{\Lambda}\right) \frac{d \mu_{\Lambda}^{i}}{d \nu_{\Lambda, y}^{t, i}}\left(x_{\Lambda}\right)
$$

Using the reversibility of $Q_{\Lambda}^{\mu_{\Lambda}}$ (resp. $Q_{\Lambda}^{\mu_{\Lambda}^{i}, i}$ ) the first term of the right hand side is obtained as follows : for any regular bounded local function $g$,

$$
\begin{aligned}
\int g\left(x_{\Lambda}\right) \nu_{\Lambda, y}^{t}\left(d x_{\Lambda}\right) & =\int g\left(\left(X_{\Lambda}(t)\right) Q_{\Lambda}^{\nu_{\Lambda, y}}(d X)\right. \\
& =\iint g\left(\left(X_{\Lambda}(t)\right) \frac{d \nu_{\Lambda, y}}{d \mu_{\Lambda}}\left(X_{\Lambda}(0)\right) Q_{\Lambda}^{\mu_{\Lambda}}(d X)\right. \\
& =\iint g\left(\left(X_{\Lambda}(0)\right) \frac{d \nu_{\Lambda, y}}{d \mu_{\Lambda}}\left(X_{\Lambda}(t)\right) Q_{\Lambda}^{\mu_{\Lambda}}(d X)\right. \\
& =\int g\left(x_{\Lambda}\right) \int \frac{d \nu_{\Lambda, y}}{d \mu_{\Lambda}}\left(X_{\Lambda}(t)\right) Q_{\Lambda}^{x_{\Lambda}}(d X) \mu_{\Lambda}\left(d x_{\Lambda}\right) .
\end{aligned}
$$

Then

$$
\frac{d \nu_{\Lambda, y}^{t}}{d \mu_{\Lambda}}\left(x_{\Lambda}\right)=E_{Q_{\Lambda}^{x_{\Lambda}}}\left(\frac{d \nu_{\Lambda, y}}{d \mu_{\Lambda}}\left(X_{\Lambda}(t)\right)\right)
$$


Doing a similar computation for the decoupled dynamics one obtains

$$
\begin{aligned}
\frac{d \nu_{\Lambda, y}^{t}}{d \nu_{\Lambda, y}^{t, i}}\left(x_{\Lambda}\right) & =E_{Q_{\Lambda}^{x_{\Lambda}}}\left(e^{f_{\Lambda, y}\left(X_{\Lambda}(t)\right)}\right) e^{-h_{i, \Lambda}\left(x_{\Lambda}\right)} E_{Q_{\Lambda, i}^{x_{\Lambda}}}\left(e^{f_{\Lambda \backslash i, y}\left(X_{\Lambda}(t)\right)}\right)^{-1} \\
& =e^{-h_{i, \Lambda}\left(x_{\Lambda}\right)+\varphi_{i}\left(x_{i}\right)} \frac{e^{f_{\Lambda}^{y}\left(x_{\Lambda}\right)}}{e^{f_{\Lambda \backslash i, y}\left(x_{\Lambda}\right)}} \frac{E_{Q_{\Lambda}^{x_{\Lambda}}}\left(e^{f_{\Lambda, y}\left(X_{\Lambda}(t)\right)-f_{\Lambda, y}\left(x_{\Lambda}\right)}\right)}{E_{Q_{\Lambda, i}^{x_{\Lambda}}}\left(e^{f_{\Lambda \backslash i, y}\left(X_{\Lambda}(t)\right)-f_{\Lambda \backslash i, y}\left(x_{\Lambda}\right)}\right)}
\end{aligned}
$$

which is the same as the expression (13).

Let us first remark that, since $\tilde{\varphi}$ is of finite range, the expression $e^{-\tilde{h}_{i}\left(x_{\Lambda} y_{\Lambda^{c}}^{\Lambda}\right)}$ does not depend on $y$ and on $\Lambda$ for $\Lambda$ large enough. We will now prove, using cluster expansions, that the last ratio in (13) is a function of $x$ indexed by $\Lambda$ which converges uniformly in $y$ when $\Lambda$ tends to $\mathbb{Z}^{d}$.

Thanks to Girsanov theorem, the probability measures $Q_{\Lambda}^{x_{\Lambda}}$ and $Q_{\Lambda, i}^{x_{\Lambda}}$ have a Gibbs representation on the path space $\mathcal{C}\left(\mathbb{R}^{+}, \mathbb{R}\right)^{\Lambda}$, that is, if one restricts them to the the canonical filtration at any time $t$ they have both an explicit density with respect to the Wiener measure with deterministic initial condition $x_{\Lambda}$, denoted by $\otimes_{i \in \Lambda} \rho^{x_{i}}$. The density of $Q_{\Lambda}^{x_{\Lambda}}$ is the following :

$$
F_{\Lambda}\left(X_{\Lambda}\right)=\exp \sum_{i \in \Lambda}\left(\int_{0}^{t}-\frac{1}{2} \nabla_{i} h_{i, \Lambda}\left(X_{\Lambda}(s)\right) d X_{i}(s)-\frac{1}{2} \int_{0}^{t} \frac{1}{4}\left(\nabla_{i} h_{i, \Lambda}\right)^{2}\left(X_{\Lambda}(s)\right) d s\right),
$$

which becomes, using Ito formula,

$$
\begin{aligned}
& F_{\Lambda}\left(X_{\Lambda}\right) \\
= & \exp \left(-\frac{1}{2} h_{\Lambda, \emptyset}\left(X_{\Lambda}(t)\right)+\frac{1}{2} h_{\Lambda, \emptyset}\left(X_{\Lambda}(0)\right)+\sum_{i \in \Lambda} \int_{0}^{t}\left(\frac{1}{4} \Delta_{i} h_{\Lambda, \emptyset}-\frac{1}{8}\left(\nabla_{i} h_{\Lambda, \emptyset}\right)^{2}\right)\left(X_{\Lambda}(s)\right) d s\right) \\
= & \exp \left(-\sum_{A \subset \Lambda} \Phi_{A}\left(X_{\Lambda}\right)\right),
\end{aligned}
$$

with

$$
\begin{aligned}
\Phi_{A}\left(X_{\Lambda}\right) & =\frac{1}{2} \varphi_{A}\left(X_{\Lambda}(t)\right)-\frac{1}{2} \varphi_{A}\left(X_{\Lambda}(0)\right) \\
& -\int_{0}^{t}\left(\frac{1}{4} \sum_{j \in A} \Delta_{j} \varphi_{A}\left(X_{\Lambda}(s)\right)-\frac{1}{8} \sum_{\substack{B, C \subset A \\
B \cup C=A \\
B \cap C \neq \emptyset}} \sum_{j \in B \cap C} \nabla_{j} \varphi_{B}\left(X_{\Lambda}(s)\right) \nabla_{j} \varphi_{C}\left(X_{\Lambda}(s)\right)\right) d s .
\end{aligned}
$$

The family $\Phi=\left(\Phi_{A}\right)_{A \subset \mathbb{Z}^{d}}$ is an interaction potential on $\Omega$; since $\varphi$ is of finite range (FR), $\Phi$ is of finite range too. Denoting by $H$ the hamiltonian function associated to $\Phi$, we then obtained that, for any $\Lambda \subset \mathbb{Z}^{d}$ and $i \in \Lambda$, on the events depending only on times between 0 and $t$,

$$
Q_{\Lambda}^{x_{\Lambda}}\left(d X_{\Lambda}\right)=e^{-H_{\Lambda, \emptyset}\left(X_{\Lambda}\right)} \otimes_{j \in \Lambda} \rho^{x_{j}}\left(d X_{j}\right) .
$$

In the same way, one proves that

$$
Q_{\Lambda, i}^{x_{\Lambda}}\left(d X_{\Lambda}\right)=e^{-H_{\Lambda \backslash i, \emptyset}\left(X_{\Lambda \backslash i}\right)} \otimes_{j \in \Lambda \backslash i} \rho^{x_{j}}\left(d X_{j}\right) \otimes \rho^{x_{i}}\left(d X_{i}\right) .
$$

Let us describe some properties of the interaction potential $\Phi$. 
Lemma 4 There exists a constant $C>0$ independent of the time $t$ such that for any $X \in \Omega$ and any $A \subset \mathbb{Z}^{d}$

$$
\left|\Phi_{A}(X)\right| \leq C\left(t+\sup _{j \in A}\left|X_{j}(t)-X_{j}(0)\right|\right) .
$$

\section{Proof :}

It is clear, due to the equality (15) and the assumptions given on $\varphi$.

We can now expend the terms $E_{Q_{\Lambda}^{x_{\Lambda}}}\left(e^{f_{\Lambda, y}\left(X_{\Lambda}(t)\right)-f_{\Lambda, y}\left(x_{\Lambda}\right)}\right)$ and $E_{Q_{\Lambda, i}^{x_{\Lambda}}}\left(e^{f_{\Lambda \backslash i, y}\left(X_{\Lambda}(t)\right)-f_{\Lambda \backslash i, y}\left(x_{\Lambda}\right)}\right)$. We give the detailed computations only for the first expansion, since the second one is obtained in a similar way.

$$
E_{Q_{\Lambda}^{x_{\Lambda}}}\left(e^{f_{\Lambda, y}\left(X_{\Lambda}(t)\right)-f_{\Lambda, y}\left(x_{\Lambda}\right)}\right)=E_{\otimes_{j \in \Lambda} \rho^{x_{j}}}\left(\exp \left(-\sum_{A \subset \Lambda} \Psi_{A}^{y, \Lambda}\left(X_{\Lambda}\right)\right)\right)
$$

where $\Psi^{y, \Lambda}$ is the following interaction potential on $\mathcal{C}([0, T], \mathbb{R})^{\Lambda}$ :

$$
\Psi_{A}^{y, \Lambda}(X)=\Phi_{A}(X)-\varphi_{A}(X(t))+\varphi_{A}(X(0))+\sum_{\substack{B \subset \mathbb{Z}^{d} \\ B \cap \Lambda=A}}\left(\tilde{\varphi}_{B}\left(X_{\Lambda}(t) y_{\Lambda^{c}}\right)-\tilde{\varphi}_{B}\left(X_{\Lambda}(0) y_{\Lambda^{c}}\right)\right) \cdot(19
$$

We also denote by $\Psi$ the interaction potential on $\mathcal{C}([0, T], \mathbb{R})^{\mathbb{Z}^{d}}$ :

$$
\Psi_{A}(X)=\Phi_{A}(X)-\varphi_{A}(X(t))+\varphi_{A}(X(0))+\left(\tilde{\varphi}_{A}(X(t))-\tilde{\varphi}_{A}(X(0))\right)
$$

and immediately remark that, as soon as $\Lambda$ is large enough with respect to the index set $A, \Psi_{A}^{y, \Lambda} \equiv \Psi_{A}$.

As in the Lemma 4 , we obtain the following estimates for the interactions $\Psi^{y, \Lambda}$ and $\Psi$.

Lemma 5 There exists a constant $C>0$ independent of the time $t$ such that for any $y \in \mathbb{R}^{\mathbb{Z}^{d}}, \Lambda \subset \mathbb{Z}^{d}, X \in \Omega$ and any $A \subset \Lambda$,

$$
\left|\Psi_{A}^{y, \Lambda}(X)\right| \leq C\left(t+\sup _{j \in A}\left|X_{j}(t)-X_{j}(0)\right|\right)
$$

and

$$
\left|\Psi_{A}(X)\right| \leq C\left(t+\sup _{j \in A}\left|X_{j}(t)-X_{j}(0)\right|\right)
$$

\section{Proof :}

It is a direct consequence of Lemma 4 and the assumptions given on $\varphi$ and $\tilde{\varphi}$. The uniformity of the first upperbound with respect to $y$ and $\Lambda$ is then clear.

Let us now introduce the main notations and tools we need for the cluster representation. Let $N \in \mathbb{N}$ large enough is such a way that for $\# A>N, \Psi_{A}^{y, \Lambda} \equiv 0$. Such a number $N$ exists since $\Psi_{A}^{y, \Lambda}$ is of finite range uniformly in $y$ and $\Lambda$. Let $\mathcal{V}$ a finite subset of $\mathbb{Z}^{d}$ such that for any $A \subset \mathbb{Z}^{d}$ with $\Psi_{A}^{y, \Lambda} \neq 0$ then $A \subset \cap_{j \in A}(\mathcal{V}+j)$.

Let us define a subclass of finite volumes in $\mathbb{Z}^{d}$ :

$$
\mathcal{D}=\left\{A \subset \mathbb{Z}^{d}: 1 \leq \# A \leq N \text { and } A \subset \cap_{j \in A}(\mathcal{V}+j)\right\}
$$


A finite set $\gamma=\left\{A_{1}, A_{2}, \ldots, A_{n}\right\}, n \geq 1$, of elements of $\mathcal{D}$ is a cluster. It is called connected if for any $A_{i}, A_{j} \in \gamma$, there exists a sequence $i=i_{1}, i_{2}, \ldots, i_{m}=j$ such that $A_{i_{1}} \cap A_{i_{2}} \neq \emptyset$, $A_{i_{2}} \cap A_{i_{3}} \neq \emptyset, \ldots, A_{i_{m-1}} \cap A_{i_{m}} \neq \emptyset$. We call support of the cluster $\gamma$ the subset of $\mathbb{Z}^{d}$ equal to $\bigcup_{l=1}^{n} A_{l}$ and denote it by $\operatorname{supp}(\gamma)$. The entire number $|\gamma|$ is the cardinality of the support of $\gamma$.

We denote by $\mathcal{A}$ the set of connected clusters and $\mathcal{A}_{\Lambda}$ the subset of $\mathcal{A}$ which contains the clusters whose supports are included in $\Lambda$. A collection of clusters $\gamma_{1}, \gamma_{2}, \ldots, \gamma_{n}$ is called compatible if their associated supports are disjoint. We denote by $\mathcal{L}_{\Lambda}$ the set of all compatible collections of non empty clusters belonging to $\mathcal{A}_{\Lambda}$.

We can now start the expansion of the expression (18).

$$
\begin{aligned}
& E_{Q_{\Lambda}^{x_{\Lambda}}}\left(e^{f_{\Lambda, y}\left(X_{\Lambda}(t)\right)-f_{\Lambda, y}\left(x_{\Lambda}\right)}\right) \\
= & E_{\otimes_{j \in \Lambda} \rho^{x_{j}}}\left(\prod_{A \subset \Lambda}\left(e^{-\Psi_{A}^{y, \Lambda}\left(X_{\Lambda}\right)}-1+1\right)\right) \\
= & E_{\otimes_{j \in \Lambda} \rho^{x_{j}}}\left(1+\sum_{n=1}^{\infty} \sum_{\left\{\gamma_{1}, \ldots \gamma_{n}\right\} \in \mathcal{L}_{\Lambda}} \mathcal{K}^{y, \Lambda}\left(\gamma_{1}\right)(X) \mathcal{K}^{y, \Lambda}\left(\gamma_{2}\right)(X) \ldots \mathcal{K}^{y, \Lambda}\left(\gamma_{n}\right)(X)\right),
\end{aligned}
$$

where

$$
\mathcal{K}^{y, \Lambda}(\gamma)(X)=\prod_{A \in \gamma}\left(e^{-\Psi_{A}^{y, \Lambda}\left(X_{\Lambda}\right)}-1\right)
$$

We then obtain the below cluster decomposition :

$$
E_{Q_{\Lambda}^{x_{\Lambda}}}\left(e^{f_{\Lambda, y}\left(X_{\Lambda}(t)\right)-f_{\Lambda, y}\left(x_{\Lambda}\right)}\right)=1+\sum_{n=1}^{\infty} \sum_{\left\{\gamma_{1}, \ldots \gamma_{n}\right\} \in \mathcal{L}_{\Lambda}} K_{x}^{y, \Lambda}\left(\gamma_{1}\right) K_{x}^{y, \Lambda}\left(\gamma_{2}\right) \ldots K_{x}^{y, \Lambda}\left(\gamma_{n}\right)
$$

where

$$
K_{x}^{y, \Lambda}(\gamma)=E_{\otimes_{j \in \mathbb{Z}^{d}} \rho^{x_{j}}}\left(\mathcal{K}^{y, \Lambda}(\gamma)(X)\right)
$$

In a similar way, we obtain for any $i \in \Lambda$ :

$$
E_{Q_{\Lambda, i}^{x_{\Lambda}}}\left(e^{f_{\Lambda \backslash i, y}\left(X_{\Lambda}(t)\right)-f_{\Lambda \backslash i, y}\left(x_{\Lambda}\right)}\right)=1+\sum_{n=1}^{\infty} \sum_{\left\{\gamma_{1}, \ldots \gamma_{n}\right\} \in \mathcal{L}_{\Lambda \backslash i}} K_{x}^{y, \Lambda}\left(\gamma_{1}\right) K_{x}^{y, \Lambda}\left(\gamma_{2}\right) \ldots K_{x}^{y, \Lambda}\left(\gamma_{n}\right) .
$$

Let also define the coefficients related to the interaction $\Psi$ (instead of $\Psi^{y, \Lambda}$ ) by :

$$
\mathcal{K}(\gamma)(X)=\prod_{A \in \gamma}\left(e^{-\Psi_{A}(X)}-1\right), \quad K_{x}(\gamma)=E_{\otimes_{j \in \mathbb{Z}^{d}} \rho^{x_{j}}}(\mathcal{K}(\gamma)) .
$$

In the next lemma, we provide estimates for the weight of the clusters (uniformly in $x, y$ and $\Lambda$ ).

Lemma 6 There exists some strictly positive constant $\lambda(t)$ which tends to 0 as $t$ goes to 0 such that, for any $x \in \mathbb{R}^{\mathbb{Z}^{d}}$, any $y \in \mathbb{R}^{\mathbb{Z}^{d}}, \Lambda \subset \mathbb{Z}^{d}$ and any $\gamma \in \mathcal{A}$

$$
\left|K_{x}^{y, \Lambda}(\gamma)\right| \leq \lambda(t)^{|\gamma|} \text { and }\left|K_{x}(\gamma)\right| \leq \lambda(t)^{|\gamma|} .
$$




\section{Proof :}

We need to commute several times integration and products. To this aim, the following abstract integration lemma, which generalizes Hölder inequalities, is very useful. It is proved in [23] Lemma 5.2 :

Lemma 7 Let $\left(\mu_{x}\right)_{x \in \mathcal{X}}$ be a family of probability measures, each one defined on a space $\mathbf{E}_{x}$, where the elements $x$ belong to some finite set $\mathcal{X}$. Let us also define a finite family $\left(g_{k}\right)_{k}$ of functions on $\mathbf{E}_{\mathcal{X}}=\times_{x \in \mathcal{X}} \mathbf{E}_{x}$ such that each $g_{k}$ is $\mathcal{X}_{k}$-local for a certain $\mathcal{X}_{k} \subset \mathcal{X}$, in the sense that

$$
g_{k}(e)=g_{k}\left(e_{\mathcal{X}_{k}}\right), \text { for } e=\left(e_{x}\right)_{x \in \mathcal{X}} \in \mathbf{E}_{\mathcal{X}} .
$$

Let $\rho_{k}>1$ be numbers satisfying the following conditions :

$$
\forall x \in \mathcal{X}, \sum_{\mathcal{X}_{k} \ni x} \frac{1}{\rho_{k}} \leq 1 .
$$

Then

$$
\left|\int_{\mathbf{E}_{\mathcal{X}}} \prod_{k} g_{k} \otimes_{x \in \mathcal{X}} d \mu_{x}\right| \leq \prod_{k}\left(\int_{\mathbf{E}_{\mathcal{X}_{k}}}\left|g_{k}\right|^{\rho_{k}} \otimes_{x \in \mathcal{X}_{k}} d \mu_{x}\right)^{1 / \rho_{k}} .
$$

Let $\gamma=\left\{A_{1}, A_{2}, \ldots, A_{n}\right\} \in \mathcal{A}$; we apply Lemma 7 with $\mathcal{X}=\operatorname{supp}(\gamma), \mathcal{X}_{k}=A_{k}, g_{k}=$ $e^{-\Psi_{A_{k}}^{y, \Lambda}}-1$ and $\rho_{k}=\frac{1}{\# \mathcal{V}}$ for all $k$. We get

$$
\left|K_{x}^{y, \Lambda}(\gamma)\right| \leq \prod_{k=1}^{n} E_{\otimes_{j \in A_{k}} \rho^{x_{j}}}\left(\left|e^{-\Psi_{A_{k}}^{y, \Lambda}}-1\right|^{\rho_{k}}\right)^{\# \mathcal{V}}
$$

Using the bound of Lemma 5, we obtain

$$
\left|K_{x}^{y, \Lambda}(\gamma)\right| \leq \prod_{k=1}^{n} E_{\otimes_{j \in A_{k}} \rho^{x_{j}}}\left(\left(e^{C\left(t+\sup _{j \in A_{k}}\left|X_{j}(t)-x_{j}\right|\right)}-1\right)^{\rho_{k}}\right)^{\# \mathcal{V}} .
$$

Now, due to the existence of exponential moment for the N-dimensional Brownian motion,

$$
E_{\bigotimes_{j \in A_{k}} \rho^{x_{j}}}\left(\left(e^{C\left(t+\sup _{j \in A_{k}}\left|X_{j}(t)-x_{j}\right|\right)}-1\right)^{\rho_{k}}\right) \leq \lambda(t),
$$

where the constant $\lambda(t)$ tends to 0 as $t$ goes to 0 , uniformly in $x$ and $A_{k} \in \mathcal{D}$. So, since $n / \rho_{k}=n \# \mathcal{V} \geq|\gamma|$, one obtains the first desired upperbound :

$$
\left|K_{x}^{y, \Lambda}(\gamma)\right| \leq \lambda(t)^{|\gamma|}
$$

The second upperbound is then obvious.

One can then deduce from Lemma 6 the following upper bound : for any cluster $\gamma \in \mathcal{A}$ and for $t$ small enough,

$$
\sup _{x, y \in \mathbb{R}^{\mathbb{Z}^{d}}} \sup _{\Lambda \subset \mathbb{Z}^{d}} \sum_{\substack{\gamma^{\prime} \in \mathcal{A}: \\ \operatorname{supp}(\gamma) \cap \operatorname{supp}\left(\gamma^{\prime}\right) \neq \emptyset}}\left|K_{x}^{y, \Lambda}\left(\gamma^{\prime}\right)\right| e^{\left|\gamma^{\prime}\right|} \leq|\gamma| .
$$


So, following Kotecký and Preiss (cf [16] page 492), we can expand for $t$ small enough the logarithmus of both, denominator and numerator of the ratio in (13):

$$
\begin{aligned}
& \ln \left(E_{Q_{\Lambda}^{x_{\Lambda}}}\left(e^{f_{\Lambda, y}\left(X_{\Lambda}(t)\right)-f_{\Lambda, y}\left(x_{\Lambda}\right)}\right)\right) \\
= & \ln \left(1+\sum_{n=1}^{\infty} \sum_{\left\{\gamma_{1}, \ldots \gamma_{n}\right\} \in \mathcal{L}_{\Lambda}} K_{x}^{y, \Lambda}\left(\gamma_{1}\right) K_{x}^{y, \Lambda}\left(\gamma_{2}\right) \ldots K_{x}^{y, \Lambda}\left(\gamma_{n}\right)\right) \\
= & \sum_{n=1}^{\infty} \sum_{\left\{\gamma_{1}, \ldots, \gamma_{n}\right\} \in \mathcal{M}_{\Lambda}} a\left(\gamma_{1}, \ldots, \gamma_{n}\right) K_{x}^{y, \Lambda}\left(\gamma_{1}\right) \ldots K_{x}^{y, \Lambda}\left(\gamma_{n}\right),
\end{aligned}
$$

and

$$
\begin{aligned}
& \ln \left(E_{Q_{\Lambda, i}^{x_{\Lambda}}}\left(e^{f_{\Lambda \backslash i, y}\left(X_{\Lambda}(t)\right)-f_{\Lambda \backslash i, y}\left(x_{\Lambda}\right)}\right)\right) \\
= & \ln \left(1+\sum_{n=1}^{\infty} \sum_{\left\{\gamma_{1}, \ldots \gamma_{n}\right\} \in \mathcal{L}_{\Lambda \backslash i}} K_{x}^{y, \Lambda}\left(\gamma_{1}\right) K_{x}^{y, \Lambda}\left(\gamma_{2}\right) \ldots K_{x}^{y, \Lambda}\left(\gamma_{n}\right)\right) \\
= & \sum_{n=1}^{\infty} \sum_{\left\{\gamma_{1}, \ldots, \gamma_{n}\right\} \in \mathcal{M}_{\Lambda \backslash i}} a\left(\gamma_{1}, \ldots, \gamma_{n}\right) K_{x}^{y, \Lambda}\left(\gamma_{1}\right) \ldots K_{x}^{y, \Lambda}\left(\gamma_{n}\right),
\end{aligned}
$$

where $\mathcal{M}_{\Lambda}$ is the set of collections of clusters $\gamma_{1}, \ldots, \gamma_{n} \in \mathcal{A}_{\Lambda}$ such that their union is also in $\mathcal{A}_{\Lambda}$, that is the union is connected too.

The logarithmus of the ratio is equal to the difference of the logarithma (26) and (27). It has then the following expansion for $t$ small :

$$
\sum_{n=1}^{\infty} \sum_{\substack{\left\{\gamma_{1}, \ldots, \gamma_{n}\right\} \in \mathcal{M}_{\Lambda}: \\ \operatorname{supp}\left(\cup_{j} \gamma_{j}\right) \ni i}} a\left(\gamma_{1}, \ldots, \gamma_{n}\right) K_{x}^{y, \Lambda}\left(\gamma_{1}\right) \ldots K_{x}^{y, \Lambda}\left(\gamma_{n}\right) .
$$

Since the inequality (25) holds uniformly in $x, y$ and $\Lambda$, following [16] (see also [1] or [21]), we conclude that the series (28) converges uniformly in $x, y$ and $\Lambda$. Moreover, for any cluster $\gamma, K_{x}^{y, \Lambda}(\gamma)$ tends to $K_{x}(\gamma)$ as $\Lambda$ goes to $\mathbb{Z}^{d}$; using Lebesgue dominated convergence theorem, we conclude that $\frac{d \nu_{\Lambda, y}^{t}}{d \nu_{\Lambda, y}^{t, i}}\left(x_{\Lambda}\right)$ converges uniformly in $x, y$ towards $e^{-\tilde{h}_{i}(x)} e^{G_{i}(x)}$ where

$$
G_{i}(x)=\sum_{n=1}^{\infty} \sum_{\substack{\left\{\gamma_{1}, \ldots, \gamma_{n}\right\} \in \mathcal{M}_{\mathbb{Z} d}: \\ \operatorname{supp}\left(\cup_{j} \gamma_{j}\right) \ni i}} a\left(\gamma_{1}, \ldots, \gamma_{n}\right) K_{x}\left(\gamma_{1}\right) \ldots K_{x}\left(\gamma_{n}\right)
$$

We are now able to complete the proof of Theorem 1.

From Lemma 2, for each regular local bounded function $g$ from $\mathbb{R}^{\Delta}$ into $\mathbb{R}$, we have

$$
\begin{aligned}
\int_{\mathbb{R}^{\mathbb{Z}^{d}}} g\left(x_{\Delta}\right) \nu^{t}(d x) & =\lim _{\Lambda \rightarrow \mathbb{Z}^{d}} \int_{\mathbb{R}^{\mathbb{Z}^{d}}} g\left(x_{\Delta}\right) \nu_{\Lambda, y}^{t}\left(d x_{\Lambda}\right) \\
& =\lim _{\Lambda \rightarrow \mathbb{Z}^{d}} \int_{\mathbb{R}^{\mathbb{Z}^{d}}} g\left(x_{\Delta}\right) \frac{d \nu_{\Lambda, y}^{t}}{d \nu_{\Lambda, y}^{t, i}\left(x_{\Lambda}\right) \nu_{\Lambda, y}^{t, i}\left(d x_{\Lambda}\right)} \\
& =\int_{\mathbb{R}^{\mathbb{Z}^{d}}} g\left(x_{\Delta}\right) e^{-\tilde{h}_{i}(x)+G_{i}(x)} \nu^{t, i}(d x) .
\end{aligned}
$$


Thus, on $\mathbb{R}^{\mathbb{Z}^{d}}$, the probability measures $\nu^{t}(d x)$ and $e^{-\tilde{h}_{i}(x)+G_{i}(x)} \nu^{t, i}(d x)$ coincide for each $i \in \mathbb{Z}^{d}$. Furthermore, since each $\nu_{\Lambda, y}^{t, i}$ is the law at time $t$ of a decoupled dynamics with decoupled initial condition, it is a product measure on $\mathbb{R}^{\Lambda \backslash i} \times \mathbb{R}^{\{i\}}$ whose projection on the $i^{t h}$-coordinate is exactly the Lebesgue measure. It implies that their infinite volume limit $\nu^{t, i}$ is a product measure on $\mathbb{R}^{\mathbb{Z}^{d} \backslash i} \times \mathbb{R}^{\{i\}}$, whose projection on the $i^{t h}$-coordinate is the Lebesgue measure too. Then, $\nu^{t}$ is a Gibbs measure associated to Lebesgue measure as reference measure and to the interaction $\varphi^{t}$ given by :

$$
\varphi_{A}^{t}(x)=\tilde{\varphi}_{A}(x)-\sum_{n=1}^{\infty} \sum_{\substack{\left\{\gamma_{1}, \ldots, \gamma_{n}\right\} \in \mathcal{M}_{A} \\ \operatorname{supp}\left(\cup_{j} \gamma_{j}\right)=A}} a\left(\gamma_{1}, \ldots, \gamma_{n}\right) K_{x}\left(\gamma_{1}\right) \ldots K_{x}\left(\gamma_{n}\right)
$$

This interaction potential is an explicit small perturbation of the initial interaction $\tilde{\varphi}$. The proof of Theorem 1 is now completed in the case $\nu \in \operatorname{ex} \mathcal{G}(\tilde{\varphi}, d x)$.

on the other hand, due to Lemma $1, \nu$ can be represented as a mixture $\nu=\int \nu_{\theta} m(d \theta)$, where $\nu_{\theta}$ are elements of ex $\mathcal{G}(\tilde{\varphi}, d x)$. Therefore, $\nu^{t}=Q^{\int \nu_{\theta} m(d \theta)} \circ X(t)^{-1}=\int\left(Q^{\nu_{\theta}} \circ\right.$ $\left.X(t)^{-1}\right) m(d \theta)=\int \nu_{\theta}^{t} m(d \theta)$. Since we just proved that $\nu_{\theta}^{t} \in \mathcal{G}\left(\varphi^{t}, d x\right)$, this implies that $\nu^{t} \in \mathcal{G}\left(\varphi^{t}, d x\right)$ too.

Proof of Remark 1 :

It is done similarly as in [26] page 71. For any $n \geq 1$ and $y \in \mathbb{R}^{\mathbb{Z}^{d}}$ we first prove that $\int_{\mathbb{R}^{\Lambda_{n}}}\|x\|_{\gamma} \nu_{\Lambda_{n}, y}(d x)$ is bounded uniformly in $n$ and $y$. For $i \in \Lambda_{n}$, by integration by parts,

$$
\tilde{Z}_{\Lambda_{n}, y}=\int_{\mathbb{R}^{\Lambda_{n}}} \exp \left(-\tilde{h}_{\Lambda_{n}, \mathbb{Z}^{d}}(x, y)\right) d x_{\Lambda_{n}}=\int x_{i} \nabla_{i} \tilde{h}_{\Lambda_{n}, \mathbb{Z}^{d}}(x, y) \exp \left(-\tilde{h}_{\Lambda_{n}, \mathbb{Z}^{d}}(x, y)\right) d x_{\Lambda_{n}} .
$$

Thus $\int x_{i} \nabla_{i} \tilde{h}_{\Lambda_{n}, \mathbb{Z}^{d}}(x, y) \nu_{\Lambda_{n}, y}\left(d x_{\Lambda_{n}}\right)=1$. But

$$
\begin{aligned}
x_{i} \nabla_{i} \tilde{h}_{\Lambda_{n}, \mathbb{Z}^{d}}(x, y) & =x_{i} \nabla_{i} \tilde{\varphi}_{i}\left(x_{i}\right)+\sum_{\substack{\Lambda \ni i \\
\# \Lambda>1}} x_{i} \nabla_{i} \tilde{\varphi}_{\Lambda}\left(x_{\Lambda_{n}} y_{\Lambda_{n}^{c}}\right) \\
& \geq a\left|x_{i}\right|-b-\sum_{\substack{\Lambda \ni i \\
\# \Lambda>1}}\left|x_{i}\right|\left\|\nabla_{i} \tilde{\varphi}_{\Lambda}\right\|_{\infty} \\
& \geq\left(a-\sum_{\substack{\Lambda \ni i \\
\# \Lambda>1}}\left\|\nabla_{i} \tilde{\varphi}_{\Lambda}\right\|_{\infty}\right)\left|x_{i}\right|-b .
\end{aligned}
$$

Thus, for some $a^{\prime}>0, \int\left(a^{\prime}\left|x_{i}\right|-b\right) \nu_{\Lambda_{n}, y}\left(d x_{\Lambda_{n}}\right) \leq 1$ which implies that there exists a constant $c>0$ independent of $n$ and $y$ such that $\int\left|x_{i}\right| \nu_{\Lambda_{n}, y}\left(d x_{\Lambda_{n}}\right) \leq c$. This bound remains valid for the integral under $\nu$, and then

$$
\int\|x\|_{\gamma} \nu(d x)=\int \sum_{i}\left|x_{i}\right| e^{-\alpha|i|} \nu(d x) \leq c \sum_{i} e^{-\alpha|i|}<+\infty .
$$




\section{Small dynamical interactions}

Let us now consider infinite-dimensional gradient dynamics where the dynamical interaction is small. Since the self-interaction does not need to be small (in the contrary) we divide the dynamical interaction into two parts as follows $U+\beta \varphi$ where $U$ is the selfinteraction and $\beta>0$ is a small parameter. We then consider the following dynamics:

$$
\left\{\begin{array}{l}
d X_{i}(t)=d B_{i}(t)-\frac{1}{2} U^{\prime}\left(X_{i}(t)\right) d t-\frac{\beta}{2} \nabla_{i} h_{i}(X(t)) d t, i \in \mathbb{Z}^{d}, t \geq 0 \\
X(0) \simeq \nu
\end{array}\right.
$$

This dynamics is a small perturbation of a free system, which is furthermore supposed to have nice ergodic properties, in such a way that its behavior for large times is close to the stationary one.

The self potential $U$, supposed to be a $\mathcal{C}^{2}$-function, is called ultracontractive if the semi-group associated to the one-dimensional free dynamics $d x(t)=d B(t)-\frac{1}{2} U^{\prime}(x(t)) d t$, where $B$ is a real-valued Brownian motion, is ultracontractive. We denote by $m$ the unique initial distribution on $\mathbb{R}$ which makes the process $x(\cdot)$ stationary; one has

$$
m(d \xi)=\frac{1}{Z} e^{-U(\xi)} d \xi, \quad \xi \in \mathbb{R} .
$$

Let us notice that there exists in the literature several types of conditions which imply the ultracontractivity of $U$. Let us cite one set which is useful (cf. Theorem 1.4 in [15]) :

$$
U^{\prime \prime}-\frac{1}{2}\left(U^{\prime}\right)^{2} \text { is bounded from above, } 0<\underline{\lim }_{|\xi| \rightarrow \infty} U^{\prime \prime}(\xi) \text { and } \int^{\infty} \frac{1}{U^{\prime}(\xi)} d \xi<+\infty .
$$

A typical example of such self-potential is given by $U(\xi)=|\xi|^{s+2}$ for some $s>0$.

In the previous section, no particular assumption was given on the set of Gibbs measures $\mathcal{G}(\tilde{\varphi}, d x)$, which contains the initial distribution $\nu$. Thus $\mathcal{G}(\tilde{\varphi}, d x)$ could be a singleton or it could have more than one element (phase transition). In the contrary, in this section, to control the evolution of the interaction at each time $t$ we use techniques involving Dobrushin's uniqueness condition, and therefore, we should suppose that the initial measure $\nu$ is the unique Gibbs measure associated to some interaction $\tilde{\varphi}: \mathcal{G}(\tilde{\varphi}, m)=\{\nu\}$.

Let us now introduce two definitions.

We say that an interaction $\phi$ on $\mathbb{R}^{\mathbb{Z}^{d}}$ satisfies the strong Dobrushin's condition if it is absolutely summable and if the following inequality holds :

$$
\sup _{i \in \mathbb{Z}^{d}} \sum_{\Lambda \ni i}(\# \Lambda-1) \sup _{x, y \in \mathbb{R}^{\Lambda}}\left|\phi_{\Lambda}(x)-\phi_{\Lambda}(y)\right|<2 .
$$

In [8] such an interaction is called a "high temperature interaction". It is well known that if an interaction $\phi$ satisfies (SDC), then it satisfies the Dobrushin's uniqueness condition which implies that $\mathcal{G}(\phi, m)$ contains at most one element (cf. for example [13], Proposition (8.8)).

We can now state our result. 
Theorem 2 Let $Q^{\nu}$ be the law on $\Omega$ of the infinite-dimensional diffusion solution of (31) where $\nu \in \mathcal{G}(\tilde{\varphi}, m)$ with support included in $l^{1}(\gamma)$, with $\gamma=\left(e^{-\alpha|i|}\right)_{i \in \mathbb{Z}^{d}}, \alpha>0$. Let us moreover suppose that

- the self-potential $U$ is ultracontractive,

- the initial interaction $\tilde{\varphi}$ satisfies (SDC), and

- the dynamical interaction $\varphi$ is of finite range (FR), regular bounded (RB) and satisfies the following assumption

$$
\sup _{\Lambda \subset \mathbb{Z}^{d}} \sup _{i \in \Lambda} \sup _{x \in \mathbb{R}^{\Lambda}}\left|U^{\prime}\left(x_{i}\right) \cdot \nabla_{i} \varphi_{\Lambda}(x)\right|<+\infty .
$$

Then, there exists $\beta_{0}>0$ depending only on $\tilde{\varphi}$ and $\varphi$ such that, for any $\beta \leq \beta_{0}$ and for all $t \geq 0$,

$$
\nu^{t}=Q^{\nu} \circ X(t)^{-1} \in \mathcal{G}\left(\varphi^{t}, m\right)
$$

where $\varphi^{t}$ is an absolutely summable (AS) interaction.

Condition (32) is a balance condition between the self-potential $\mathrm{U}$ and the dynamical potential $\varphi$. This is satisfied for example for any potential $\varphi$ which is constant at infinity.

\section{Proof :}

Let us first remark that for $\tilde{\varphi}$ small enough, we could use similar techniques as in the proof of Theorem 1, writing the cluster expansion now no more with respect to the time but with respect to both small parameters $\|\tilde{\varphi}\|_{\infty}$ and $\beta$. But we want to obtain more than a perturbative result around the free stationnary case. Therefore, when $\tilde{\varphi}$ is not supposed to be close to 0 , we have to develop other techniques than before. To this aim let us introduce some more notations.

As in the last section, the infinite-dimensional dynamics (31) is obtained as limit of the following finite dimensional dynamics : for $\Lambda \subset \mathbb{Z}^{d}, \Lambda$ finite ,

$$
d X_{i}(t)=d B_{i}(t)-\frac{1}{2} U^{\prime}\left(X_{i}(t)\right) d t-\frac{\beta}{2} \nabla_{i} h_{i, \Lambda}(X(t)) d t, i \in \Lambda, t \geq 0 .
$$

For any $x, y \in \mathbb{R}^{\mathbb{Z}^{d}}, \Lambda \subset \mathbb{Z}^{d}$ and $I=[a, b]$, we use the notations :

- $Q^{\nu}$ (resp. $\left.Q^{x}\right)$ : law on $\Omega$ of the solution of (31) with initial distribution $\nu$ (resp. $\delta_{x}$ ).

- $Q_{\Lambda}^{x_{\Lambda}}$ : law on $\mathcal{C}\left(\mathbb{R}^{+}, \mathbb{R}\right)^{\Lambda}$ of the solution of (33) with initial deterministic condition $x_{\Lambda}$.

- $P^{x}$ : law on $\Omega=\mathcal{C}\left(\mathbb{R}^{+}, \mathbb{R}\right)^{\mathbb{Z}^{d}}$ of the solution of the free system $((31)$ with $\beta=0)$ and initial condition $x$; it is the infinite product of the one-dimensional free dynamics $P_{i}^{x_{i}}$, each of one having at time $t p_{t}\left(x_{i}, \cdot\right)$ as density function with respect to $m$.

- $P_{\Lambda}^{x_{\Lambda}}$ : law on $\mathcal{C}\left(\mathbb{R}^{+}, \mathbb{R}\right)^{\Lambda}$ of the solution of $(33)$ when $\beta=0$ with initial condition $x_{\Lambda}$.

- $P_{\Lambda, I}^{x, y}$ : law on $\mathcal{C}(I, \mathbb{R})^{\Lambda}$ of the solution of $(33)$ when $\beta=0$ conditioned by the initial and the final values : $X_{\Lambda}(a)=x_{\Lambda}$ et $X_{\Lambda}(b)=y_{\Lambda}$

Step 1 : Density of $Q_{\Lambda}^{x_{\Lambda}} \circ X(t)^{-1}$ with respect to $P_{\Lambda}^{x_{\Lambda}} \circ X(t)^{-1}$ on $\mathbb{R}^{\Lambda}$

Our first aim is to obtain this density as an exponential function of an uniformly convergent sum of interactions. By Girsanov theorem, $Q_{\Lambda}^{x_{\Lambda}}$ restricted to the canonical 
filtration at time $t$ is absolutely continuous with respect to $P_{\Lambda}^{x_{\Lambda}}$ with density denoted by $F_{\Lambda}$. Making similar computations as in the proof of (14), one obtains

$$
\begin{aligned}
F_{\Lambda}\left(X_{\Lambda}\right)= & \exp \left(-\frac{1}{2} \beta h_{\Lambda, \emptyset}\left(X_{\Lambda}(t)\right)+\frac{1}{2} \beta h_{\Lambda, \emptyset}\left(X_{\Lambda}(0)\right)+\sum_{i \in \Lambda} \int_{0}^{t}\left(\frac{1}{4} \beta \Delta_{i} h_{\Lambda, \emptyset}\left(X_{\Lambda}(s)\right)\right.\right. \\
& \left.\left.-\frac{1}{8} \beta^{2}\left(\nabla_{i} h_{\Lambda, \emptyset}\right)^{2}\left(X_{\Lambda}(s)\right)+\frac{1}{4} \beta U^{\prime}\left(X_{i}(s)\right) \nabla_{i} h_{\Lambda, \emptyset}\left(X_{\Lambda}(s)\right)\right) d s\right) \\
= & \exp \left(-\frac{1}{2} \beta h_{\Lambda, \emptyset}\left(X_{\Lambda}(t)\right)+\frac{1}{2} \beta h_{\Lambda, \emptyset}\left(X_{\Lambda}(0)\right)+\int_{0}^{t} \sum_{A \subset \Lambda} g_{A}(X(s)) d s\right),
\end{aligned}
$$

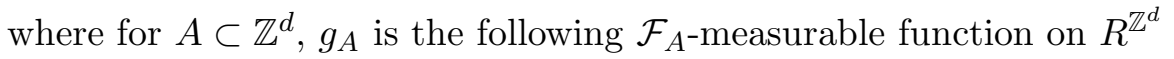

$$
g_{A}(x)=\frac{1}{4} \beta \sum_{i \in A}\left(\Delta_{i} \varphi_{A}\left(x_{A}\right)+U^{\prime}\left(x_{i}\right) \nabla_{i} \varphi_{A}\left(x_{A}\right)\right)-\frac{1}{8} \beta^{2} \sum_{\substack{B, C \subset A \\ B \cup C=A \\ B \cap C \neq \emptyset}} \sum_{i \in B \cap C} \nabla_{i} \varphi_{B}\left(x_{B}\right) \nabla_{i} \varphi_{C}\left(x_{C}\right) .
$$

Moreover, due to the assumptions on $\varphi$ and (32) there exists a constant $\tilde{C}>0$ such that

$$
\forall A \subset \mathbb{Z}^{d}, \quad\left\|g_{A}\right\|_{\infty} \leq \tilde{C} \beta .
$$

From (34), one deduces that

$$
\frac{Q_{\Lambda}^{x_{\Lambda}} \circ X(t)^{-1}}{P_{\Lambda}^{x_{\Lambda}} \circ X(t)^{-1}}\left(y_{\Lambda}\right)=e^{-\frac{1}{2} \beta\left(h_{\Lambda, \emptyset}\left(y_{\Lambda}\right)-\beta h_{\Lambda, \emptyset}\left(x_{\Lambda}\right)\right)} \mathbf{f}_{\Lambda}(x, y)
$$

with

$$
\mathbf{f}_{\Lambda}(x, y)=E_{P_{\Lambda,[0, t]}^{x, y}}\left[\exp \left(\int_{0}^{t} \sum_{A \subset \Lambda} g_{A}(X(s)) d s\right)\right]
$$

We are now looking for a cluster representation of $\mathbf{f}_{\Lambda}(x, y)$ for $\beta$ small.

We first work at the space-time level as in [3] or [22] (cf. also [14] or [20]); Clusters are then subsets of $\mathbb{Z}^{d} \times \mathbb{N}$. In Step 2 , we will project this representation at times 0 and $t$, obtaining clusters in $\mathbb{Z}^{d} \times\{0,1\}$.

Let us introduce the notations we need. Let $N \in \mathbb{N}$ large enough is such a way that for $\# A>N, g_{A} \equiv 0$. Such a number $N$ exists since $g_{A}$ is of finite range by constrution.

Let $\mathcal{V}$ a finite subset of $\mathbb{Z}^{d}$ such that for any $A \subset \mathbb{Z}^{d}$ with $g_{A} \neq 0$ then $A \subset \cap_{j \in A}(\mathcal{V}+j)$. Let us define a subclass of finite volumes in $\mathbb{Z}^{d} \times \mathbb{N}$ :

$$
\mathcal{D}=\left\{\mathbf{A}=A \times\{j, j+1\} \subset \mathbb{Z}^{d} \times \mathbb{N}: 1 \leq \# A \leq N \text { and } A \subset \cap_{i \in A}(\mathcal{V}+i)\right\} .
$$

A finite set $\gamma=\left\{\mathbf{A}_{1}, \mathbf{A}_{2}, \ldots, \mathbf{A}_{n}\right\}, n \geq 1$, of elements of $\mathcal{D}$ is a cluster. It is called connected if for any $\mathbf{A}_{i}, \mathbf{A}_{j} \in \gamma$, there exists a sequence $i=i_{1}, i_{2}, \ldots, i_{m}=j$ such that $\mathbf{A}_{i_{1}} \cap \mathbf{A}_{i_{2}} \neq \emptyset, \mathbf{A}_{i_{2}} \cap \mathbf{A}_{i_{3}} \neq \emptyset, \ldots, \mathbf{A}_{i_{m-1}} \cap \mathbf{A}_{i_{m}} \neq \emptyset$. We call support of the cluster $\gamma$ the subset of $\mathbb{Z}^{d} \times \mathbb{N}$ equal to $\bigcup_{l=1}^{n} \mathbf{A}_{l}$ and denote it by $\operatorname{supp}(\gamma)$. The entire number $|\gamma|$ is the cardinality of the support of $\gamma$.

We denote by $\mathcal{A}$ the set of connected clusters and $\mathcal{A}_{\Lambda \times[0, M]}$ the subset of $\mathcal{A}$ which contains 
the clusters whose supports are included in $\Lambda \times\{0, \cdots, M\} \subset \mathbb{Z}^{d} \times \mathbb{N}$. A collection of clusters $\gamma_{1}, \gamma_{2}, \ldots, \gamma_{n}$ is called compatible if their associated supports are disjoint. We denote by $\mathcal{L}_{\Lambda \times[0, M]}$ the set of all compatible collections of non empty clusters belonging to $\mathcal{A}_{\Lambda \times[0, M]}$.

We can now start the expansion of the expression (37).

Let $M$ be some integer which we will fix later, and $T=\frac{t}{M}$. We decompose the time interval $[0, t]$ into $M$ subintervals $I_{j}=[j T ;(j+1) T], j=0, \cdots, M-1$. We obtain, taking $x_{0}=x$,

$$
\begin{aligned}
\mathbf{f}_{\Lambda}(x, y)= & \iint \ldots \int \prod_{A \subset \Lambda} \prod_{j=0}^{M-1} \exp \left(\int_{I_{j}} g_{A}(X(s)) d s\right) \prod_{j=0}^{M-2} \prod_{i \in \Lambda} p_{T}\left(x_{i}^{(j)}, x_{i}^{(j+1)}\right) \\
& P_{\Lambda, I_{0}}^{x, x^{(1)}}(d X) P_{\Lambda, I_{1}}^{x^{(1)}, x^{(2)}}(d X) \ldots P_{\Lambda, I_{M-1}}^{x^{(M-1)}, y}(d X) m^{\otimes \Lambda}\left(d x^{(1)}\right) \ldots m^{\otimes \Lambda}\left(d x^{(M-1)}\right), \\
= & \iint \ldots \int\left(1+\sum_{n=0}^{\infty} \sum_{\left\{\gamma_{1}, \ldots, \gamma_{n}\right\} \in \mathcal{L}_{\Lambda \times[0, M]}} \mathcal{K}_{M}\left(\gamma_{1}\right)(X) \mathcal{K}_{M}\left(\gamma_{2}\right)(X) \ldots \mathcal{K}_{M}\left(\gamma_{n}\right)(X)\right) \\
& P_{\Lambda, I_{0}}^{x, x^{(1)}}(d X) P_{\Lambda, I_{1}}^{x^{(1)}, x^{(2)}}(d X) \ldots P_{\Lambda, I_{M-1}}^{x^{(M-1)}, y}(d X) m^{\otimes \Lambda}\left(d x^{(1)}\right) \ldots m^{\otimes \Lambda}\left(d x^{(M-1)}\right) .
\end{aligned}
$$

$\mathcal{K}_{M}(\gamma)$ has the following form :

$$
\begin{aligned}
\mathcal{K}_{M}(\gamma)(X)= & \prod_{\substack{\{i\} \times\{j, j+1\} \in \gamma \\
j \leq M-2}}\left(p_{T}\left(X_{i}(j T), X_{i}((j+1) T)\right) \exp \left(\int_{I_{j}} g_{i}(X(s)) d s\right)-1\right) \\
& \prod_{\substack{A \times\{j, j+1\} \in \gamma \\
\# A \geq 2 \\
j \leq M-2}}\left(\exp \left(\int_{I_{j}} g_{A}(X(s)) d s\right)-1\right) \\
& \prod_{A \times\{M-1, M\} \in \gamma}\left(\exp \left(\int_{I_{M-1}} g_{A}(X(s)) d s\right)-1\right) .
\end{aligned}
$$

We denote by $\epsilon(T)$ the fluctuation of the kernel $p_{T}$ around the equilibrium :

$$
\epsilon(T)=\sup _{a, b \in \mathbb{R}}\left|p_{T}(a, b)-1\right|
$$

Since $U$ is ultracontractive, one has

$$
\lim _{T \rightarrow \infty} \epsilon(T)=0
$$

Let us choose $\tilde{\beta}_{0}$ and $T_{0}$ such that for any $T>T_{0}$ and $\beta<\tilde{\beta}_{0},(1+\epsilon(T)) e^{\tilde{C} T \beta}-1 \leq 1$. We consider now both cases, $t \leq T_{0}$ and $t>T_{0}$ separately.

For $t \leq T_{0}$ we fix the integer $M$ equal to 1 . So $T=t$ and we only have to control $\mathcal{K}_{1}(\gamma)$, which has the following simple form :

$$
\left|\mathcal{K}_{1}(\gamma)(X)\right|=\left|\prod_{A \times\{0,1\} \in \gamma}\left(\exp \left(\int_{[0, t]} g_{A}(X(s)) d s\right)-1\right)\right| \leq\left(e^{\tilde{C} T_{0} \beta}-1\right)^{\frac{|\gamma|}{2 \# \mathcal{V}}}
$$


For $t>T_{0}$ we fix the integer $M$ equal to $[t \sqrt{\beta}]+1$, which is the smallest integer strictly larger than $t \sqrt{\beta}$; so $T=\frac{t}{[t \sqrt{\beta}]+1}$, which tends to $+\infty$ when $\beta$ vanishes. For $\beta$ small enough, we obtain

$$
\begin{aligned}
\left|\mathcal{K}_{M}(\gamma)(X)\right| & \leq\left((1+\epsilon(T)) e^{\tilde{C} T \beta}-1\right)^{\frac{|\gamma|}{2 \# \mathcal{V}}} \\
& \leq 2\left(\left(1+\epsilon\left(\frac{1}{\sqrt{\beta}}\right)\right) e^{\tilde{C} \sqrt{\beta}}-1\right)^{\frac{|\gamma|}{2 \# \mathcal{V}}} \\
& \leq \lambda(\beta)^{|\gamma|},
\end{aligned}
$$

where $\lambda(\beta)$ tends to 0 as $\beta$ goes to 0 . Coming back to $\mathbf{f}_{\Lambda}(x, y)$, we obtain

$$
\mathbf{f}_{\Lambda}(x, y)=1+\sum_{n=0}^{\infty} \sum_{\left\{\gamma_{1}, \ldots, \gamma_{n}\right\} \in \mathcal{L}_{\Lambda \times[0, M]}} \mathbf{K}_{M}^{x, y}\left(\gamma_{1}\right) \mathbf{K}_{M}^{x, y}\left(\gamma_{2}\right) \ldots \mathbf{K}_{M}^{x, y}\left(\gamma_{n}\right)
$$

where

$$
\mathbf{K}_{M}^{x, y}(\gamma)=\int \ldots \int \mathcal{K}_{M}(\gamma)(X) P_{\Lambda, I_{0}}^{x, x^{(1)}}(d X) \ldots P_{\Lambda, I_{M-1}}^{x^{(M-1)}, y}(d X) m^{\otimes \Lambda}\left(d x^{(1)}\right) \ldots m^{\otimes \Lambda}\left(d x^{(M-1)}\right),
$$

so that

$$
\sup _{x, y, t}\left|\mathbf{K}_{M}^{x, y}(\gamma)\right| \leq \lambda(\beta)^{|\gamma|} .
$$

As in the last section, this implies that for any cluster $\gamma \in \mathcal{A}$ and for $\beta$ small enough,

$$
\sup _{x, y \in \mathbb{R}^{\mathbb{Z}^{d}, t>0}} \sum_{\substack{\gamma^{\prime} \in \mathcal{A}: \\ \operatorname{supp}(\gamma) \cap \operatorname{supp}\left(\gamma^{\prime}\right) \neq \emptyset}}\left|\mathbf{K}_{M}^{x, y}\left(\gamma^{\prime}\right)\right| e^{\left|\gamma^{\prime}\right|} \leq|\gamma| .
$$

For $\beta$ small enough, following Kotecký and Preiss, one obtains the following expansion for the logarithmus of $\mathbf{f}_{\Lambda}(x, y)$ :

$$
\ln \left(\mathbf{f}_{\Lambda}(x, y)\right)=\sum_{n=0}^{\infty} \sum_{\left\{\gamma_{1}, \ldots, \gamma_{n}\right\} \in \mathcal{M}_{\Lambda \times[0, M]}} a\left(\gamma_{1}, \ldots, \gamma_{n}\right) \mathbf{K}_{M}^{x, y}\left(\gamma_{1}\right) \mathbf{K}_{M}^{x, y}\left(\gamma_{2}\right) \ldots \mathbf{K}_{M}^{x, y}\left(\gamma_{n}\right) .
$$

We now leave the space-time level and go to the level of the projections at times 0 and $t$, obtaining :

$$
\ln \left(\mathbf{f}_{\Lambda}(x, y)\right)=\sum_{\Delta \subset \Lambda} \sum_{n=0}^{\infty} \sum_{\substack{\left\{\gamma_{1}, \ldots, \gamma_{n}\right\} \in \mathcal{M}_{\Lambda \times[0, M]} \\ \operatorname{Tr}\left(\gamma_{1}, \ldots, \gamma_{n}\right)=\Delta}} a\left(\gamma_{1}, \ldots, \gamma_{n}\right) \mathbf{K}_{M}^{x, y}\left(\gamma_{1}\right) \ldots \mathbf{K}_{M}^{x, y}\left(\gamma_{n}\right),
$$

where $\operatorname{Tr}\left(\gamma_{1}, \ldots, \gamma_{n}\right)$ denotes the spatial trace of the cluster $\gamma_{1}, \ldots, \gamma_{n}$, that is the projection of its support on $\mathbb{Z}^{d}$.

Thus from (36),

$$
\frac{Q_{\Lambda}^{x} \circ X(t)^{-1}}{P_{\Lambda}^{x} \circ X(t)^{-1}}\left(y_{\Lambda}\right)=e^{-\sum_{\Delta \subset \Lambda} \Phi_{\Delta}^{\beta}(x, y)}
$$


where

$$
\begin{aligned}
\boldsymbol{\Phi}_{\Delta}^{\beta}(x, y)= & \frac{1}{2} \beta\left(\varphi_{\Delta}(y)-\varphi_{\Delta}(x)\right) \\
& -\sum_{n=0}^{\infty} \sum_{\substack{\left\{\gamma_{1}, \ldots, \gamma_{n}\right\} \in \mathcal{M}_{\Lambda \times[0, M]} \\
\operatorname{Tr}\left(\gamma_{1}, \ldots, \gamma_{n}\right)=\Delta}} a\left(\gamma_{1}, \ldots, \gamma_{n}\right) \mathbf{K}_{M}^{x, y}\left(\gamma_{1}\right) \ldots \mathbf{K}_{M}^{x, y}\left(\gamma_{n}\right) .
\end{aligned}
$$

We note some important properties of the function $\boldsymbol{\Phi}^{\beta}$.

Lemma 8 The function $\boldsymbol{\Phi}_{\Delta}^{\beta}(x, y)$ defined on $\mathbb{R}^{\mathbb{Z}^{d}} \times \mathbb{R}^{\mathbb{Z}^{d}}$ is indeed $\mathcal{F}_{\Delta} \times \mathcal{F}_{\Delta}$-measurable and satisfies

$$
\lim _{\beta \rightarrow 0} \sup _{i \in \mathbb{Z}^{d}} \sum_{\Delta \ni i}(\# \Delta-1)\left\|\Phi_{\Delta}^{\beta}\right\|_{\infty}=0 .
$$

\section{Proof :}

The measurability property of $\boldsymbol{\Phi}_{\Delta}^{\beta}$ is a consequence of the following observation : $\mathbf{K}_{M}^{x, y}(\gamma)$ depend on $x$ (resp. on $y$ ) only on $\operatorname{supp}(\gamma) \cap\left(\mathbb{Z}^{d} \times\{0\}\right) \subset \operatorname{Tr}(\gamma)=\Delta($ resp. on $\operatorname{supp}(\gamma) \cap$ $\left(\mathbb{Z}^{d} \times\{t\}\right) \subset \Delta$, so that in fact $\boldsymbol{\Phi}_{\Delta}^{\beta}(x, y)=\boldsymbol{\Phi}_{\Delta}^{\beta}\left(x_{\Delta}, y_{\Delta}\right)$.

Moreover, Kotecký and Preiss proved in [16] the following exponential decrease of $\boldsymbol{\Phi}_{\Delta}^{\beta}$ with respect to $\Delta$ : For any $a \in \mathbb{R}$, there exists $\beta_{a}>0$ such that for all $\beta \leq \beta_{a}$ one has

$$
\sup _{i \in \mathbb{Z}^{d}} \sup _{t \in \mathbb{R}^{+}} \sum_{\Delta \ni i} e^{a \# \Delta}\left\|\boldsymbol{\Phi}_{\Delta}^{\beta}\right\|_{\infty} \leq 1
$$

This implies that, uniformly in $i$ and $t$, the sum $\sum_{\Delta \ni i}(\# \Delta-1)\left\|\boldsymbol{\Phi}_{\Delta}^{\beta}\right\|_{\infty}$ converges for $\beta$ small enough. Since $\lim _{\beta \rightarrow 0}\left\|\Phi_{\Delta}^{\beta}\right\|_{\infty}=0$, we obtain the desired result.

$\underline{\text { Step } 2 \text { : Study of } \mathbf{Q}^{\nu}=Q^{\nu} \circ(X(0), X(t))^{-1} \text { on } \mathbb{R}^{\mathbb{Z}^{d} \times\{0,1\}} \text { and its Gibbsian properties }}$

In order to prove the Gibbsianness of $\nu^{t}=Q^{\nu} \circ X(t)^{-1}$, we study as intermediate step $\mathbf{Q}^{\nu}=Q^{\nu} \circ(X(0), X(t))^{-1}$, the joint projection of $Q^{\nu}$ at time 0 and $\mathrm{t}$ on the space $\mathbb{R}^{S}$, where $S$ is the so-called bi-space : $S=\mathbb{Z}^{d} \times\{0,1\}$. (In the framework of Probabilistic Cellular Automata, the idea to analyse the properties of the process on a bi-space was already powerful, cf. [28]).

Lemma 9 For $\beta$ sufficiently small $\mathbf{Q}^{\nu}$ is a Gibbs measure on $\mathbb{R}^{S}$ with reference measure $m$ and associated Hamiltonian function $\mathbf{H}$ which is defined as follows : if we denote by $\left(\Delta, \Delta^{\prime}\right)$ the subset of $S$ equal to $(\Delta \times\{0\}) \cup\left(\Delta^{\prime} \times\{1\}\right)$,

$$
\mathbf{H}_{\left(\Delta, \Delta^{\prime}\right)}(x, y)=\tilde{h}_{\Delta}(x)-\sum_{i \in \Delta \cup \Delta^{\prime}} \ln p_{t}\left(x_{i}, y_{i}\right)+\sum_{\substack{A \subset \mathbb{Z}^{d} \\ A \cap\left(\Delta \cup \Delta^{\prime}\right) \neq \emptyset}} \boldsymbol{\Phi}_{A}^{\beta}(x, y) .
$$

\section{Proof :}

Since the initial interaction $\tilde{\varphi}$ satisfies the strong Dobrushin's condition (SDC), $\nu$ is the unique element in $\mathcal{G}(\tilde{\varphi}, m)$; it can be approximated by the sequence of finite volume specifications with free boundary conditions : $\left(\nu_{\Lambda}=\frac{1}{z_{\Lambda}} \exp -\tilde{h}_{\Lambda, \emptyset} m^{\otimes \Lambda}\right)_{\Lambda \subset \mathbb{Z}^{d}}$. Let $Q_{\Lambda}^{\nu_{\Lambda}}$ be the law on $\mathcal{C}\left(\mathbb{R}^{+}, \mathbb{R}\right)^{\Lambda}$ of the solution of (33) with initial distribution $\nu_{\Lambda}$. Similarly to the 
proof of Lemma 2, for any bounded regular local functional $G$, the sequence $\int G d Q_{\Lambda}^{\nu_{\Lambda}}$ converges towards $\int G d Q^{\nu}$ when $\Lambda$ tends to $\mathbb{Z}^{d}$. In particular,

$$
\lim _{\Lambda \rightarrow \mathbb{Z}^{d}} \int G(X(0), X(t)) d Q_{\Lambda}^{\nu_{\Lambda}}=\int G(X(0), X(t)) d Q^{\nu},
$$

which means that the joint projection of $Q_{\Lambda}^{\nu_{\Lambda}}$ at times 0 and $t$ on the space $\left(\mathbb{R}^{2}\right)^{\Lambda}$ converges towards $\mathbf{Q}^{\nu}$, the joint projection of $Q^{\nu}$ at times 0 and $t$, considered as probability measure on $\left(\mathbb{R}^{2}\right)^{\mathbb{Z}^{d}}$. Now, for $\beta$ sufficiently small, using Girsanov formula and (42), it is clear that the family $\left(Q_{\Lambda}^{\nu_{\Lambda}}\right)_{\Lambda}$ is the Gibbsian specification on $\left(\mathbb{R}^{2}\right)^{\mathbb{Z}^{d}}$ with free boundary condition associated to the reference measure on $\mathbb{R}^{2} \mathbf{m}(d \xi, d \zeta)=p_{t}(\xi, \zeta) m(d \xi) m(d \zeta)$ and the interaction

$$
\Psi_{\Delta}(x, y)=\tilde{\varphi}_{\Delta}(x)+\Phi_{\Delta}^{\beta}(x, y),(x, y) \in\left(\mathbb{R}^{2}\right)^{\mathbb{Z}^{d}}, \Delta \subset \mathbb{Z}^{d} .
$$

(This function on $\left(\mathbb{R}^{2}\right)^{\mathbb{Z}^{d}}$ is indeed an interaction due to the measurability property of $\boldsymbol{\Phi}_{\Delta}^{\beta}$ proved in Lemma 8.) Since $\tilde{\varphi}$ satisfies the strong Dobrushin's condition (SDC) and, by Lemma $8, \sum_{\Delta \ni i}(\# \Delta-1)\left\|\boldsymbol{\Phi}_{\Delta}^{\beta}\right\|_{\infty}$ is as small as required for $\beta$ small enough, the interaction $\Psi$ satisfies also Condition (SDC) for $\beta$ small. This implies in particular that the set $\mathcal{G}(\Psi, \mathbf{m})$ of Gibbs measures on $\left(\mathbb{R}^{2}\right)^{\mathbb{Z}^{d}}$ contains at most one element; this element is nothing but $\mathbf{Q}^{\nu}$, limit of the free boundary specifications (see [13] Example (4.20) for the relation between free boundary conditions and usual boundary conditions.) The measure $\mathbf{Q}^{\nu}$ thus satisfies (DLR) equations with the Hamiltonian function associated to $\Psi$ and the reference measure $\mathbf{m}$. If we now consider the natural bijection between $\left(\mathbb{R}^{2}\right)^{\mathbb{Z}^{d}}$ and $\mathbb{R}^{S}, \mathbf{Q}^{\nu}$ is a measure on $\mathbb{R}^{S}$ satisfying (DLR) equations for any finite volume of the type $(\Delta, \Delta) \subset S$, with Hamiltonian function $\mathbf{H}_{(\Delta, \Delta)}$ and reference measure $m$. This is enough to deduce the desired result, since any finite volume $\left(\Delta, \Delta^{\prime}\right) \subset S$ can be included in some symmetrical volume $\left(\Delta^{\prime \prime}, \Delta^{\prime \prime}\right) \subset S$.

We now desintegrate the measure $\mathbf{Q}^{\nu}$ with respect to finite-dimensional projections at time $t$. Let us denote by $\mathbf{Q}^{\nu, y_{\Lambda^{c}}}$ the probability measure $\mathbf{Q}^{\nu}\left(\cdot \mid X_{\Lambda^{c}}(t)=y_{\Lambda^{c}}\right)$, which is defined for any finite subset $\Lambda$ of $\mathbb{Z}^{d}$, and for $\nu^{t}$-a.a. $y$. The next lemma gives a Gibbsian description of this measure. Its simple proof is omitted.

Lemma 10 The probability measure $\mathbf{Q}^{\nu, y_{\Lambda^{c}}}$, conditional law of $Q^{\nu} \circ(X(0), X(t))^{-1}$ given $\left\{X_{\Lambda^{c}}(t)=y_{\Lambda^{c}}\right\}$, is a Gibbs measure on $\mathbb{R}^{\mathbb{Z}^{d} \times\{0\} \cup \Lambda \times\{1\}}$ with reference measure $m$ and Hamiltonian function $\mathbf{H}^{y_{\Lambda}^{c}}$ defined for $\left(\Delta, \Delta^{\prime}\right) \subset \mathbb{Z}^{d} \times \Lambda$ by

$$
\mathbf{H}_{\left(\Delta, \Delta^{\prime}\right)}^{y_{\Lambda^{c}}}\left(x, z_{\Lambda}\right)=\mathbf{H}_{\left(\Delta, \Delta^{\prime}\right)}\left(x, z_{\Lambda} y_{\Lambda^{c}}\right), \quad x \in \mathbb{R}^{\mathbb{Z}^{d}}, z_{\Lambda} \in \mathbb{R}^{\Lambda} .
$$

$\mathbf{Q}^{\nu, y_{\Lambda} c}$ can be decoupled as follows :

$$
\begin{aligned}
& \mathbf{Q}^{\nu, y_{\Lambda^{c}}}\left(d x, d z_{\Lambda}\right)= \\
& \frac{1}{\mathbf{Z}_{\Lambda}\left(y_{\Lambda^{c}}\right)} \prod_{i \in \Lambda} p_{t}\left(x_{i}, z_{i}\right) \exp \left(-\sum_{\substack{A \subset \mathbb{Z}^{d} \\
A \cap \Lambda \neq \emptyset}} \boldsymbol{\Phi}_{A}^{\beta}\left(x, z_{\Lambda} y_{\Lambda^{c}}\right)\right) m^{\otimes \Lambda}\left(d z_{\Lambda}\right) \bar{Q}^{\nu, y_{\Lambda^{c}}}(d x),
\end{aligned}
$$

where $\bar{Q}^{\nu, y_{\Lambda^{c}}}$ is a probability measure on $\mathbb{R}^{\mathbb{Z}^{d}}$. Furthermore, $\overline{\mathbf{Q}}^{\nu, y_{\Lambda^{c}}}$ belongs to $\mathcal{G}\left(\bar{\Phi}^{y_{\Lambda^{c}}}, m\right)$ where the interaction $\bar{\Phi}^{y_{\Lambda^{c}}}$ is defined for $x \in \mathbb{R}^{\mathbb{Z}^{d}}$ by :

$$
\left\{\begin{array}{l}
\bar{\Phi}_{i}^{y_{\Lambda^{c}}}(x)=\tilde{\varphi}_{i}\left(x_{i}\right)-\mathbb{I}_{i \in \Lambda^{c}} \ln p_{t}\left(x_{i}, y_{i}\right), \quad i \in \mathbb{Z}^{d} \\
\bar{\Phi}_{\Delta}^{y_{\Lambda^{c}}}(x)=\tilde{\varphi}_{\Delta}(x)+\mathbb{I}_{\Delta \cap \Lambda=\emptyset} \Phi_{\Delta}^{\beta}\left(x, y_{\Lambda^{c}}\right), \text { for } \Delta \subset \mathbb{Z}^{d}, \# \Delta \geq 2 .
\end{array}\right.
$$


In the next lemma we show that uniformly (with respect to $\Lambda$ and $y$ ) the local specifications of $\bar{Q}^{\nu, y_{\Lambda^{c}}}$ converge to this Gibbs measure.

Lemma 11 For $\beta$ sufficiently small and $t>0$, for $\Lambda \subset \mathbb{Z}^{d}$ and for all $y \in \mathbb{R}^{\mathbb{Z}^{d}}$, the set $\mathcal{G}\left(\bar{\Phi}^{y_{\Lambda^{c}}}, m\right)$ contains a unique element denoted by $\bar{Q}^{\nu, y_{\Lambda^{c}}}$. Moreover, for any $\Delta \subset \mathbb{Z}^{d}$,

$$
\lim _{\Delta^{\prime} \nearrow \mathbb{Z}^{d}} \sup _{A \in \mathcal{F}_{\Delta}} \sup _{\Lambda \subset \mathbb{Z}^{d}} \sup _{y \in \mathbb{R}^{\mathbb{Z}^{d}}} \sup _{x \in \mathbb{R}^{\mathbb{Z}^{d}}}\left|\bar{Q}^{\nu, y_{\Lambda^{c}}}(A)-\bar{Q}^{\nu, y_{\Lambda^{c}}}\left(A \mid x_{\Delta^{\prime c}}\right)\right|=0 .
$$

Proof: Considering the form of the interaction potential $\bar{\Phi}^{y_{\Lambda^{c}}}$ given in (47), it is obvious, as in the proof of Lemma 9, that for $\beta$ sufficiently small, $\bar{\Phi}^{y_{\Lambda^{c}}}$ satisfies (SDC). Therefore the set $\mathcal{G}\left(\bar{\Phi}^{y_{\Lambda^{c}}}, m\right)$ contains at most one element. The strong convergence of the local specifications to the limiting Gibbs measure is a classical result, which is proved for example in [13], Theorem 8.23. The uniformity in $\Lambda$ and $y$ in (48) comes from the same uniformity obtained in the (SDC).

Remark 2 Since $\bar{Q}^{\nu, y_{\Lambda^{c}}}$ is well defined for any $y \in \mathbb{R}^{\mathbb{Z}^{d}}, \mathbf{Q}^{\nu, y_{\Lambda^{c}}}$ is also defined by (46) not only for $\nu^{t}$-a.a. $y$ but for all $y$. This is a regular version of the conditional probabilities $\mathbf{Q}^{\nu}\left(d x d y \mid y_{\Lambda^{c}}\right)$.

We now observe that, for a.a. $y_{\Lambda^{c}} \in \mathbb{R}^{\Lambda^{c}}, \nu_{t}\left(\cdot \mid y_{\Lambda^{c}}\right)$ is the marginal on $\mathbb{R}^{\mathbb{Z}^{d}}$ of $\mathbf{Q}^{\nu, y_{\Lambda^{c}}}$. This means that, for any regular bounded function $g$ on $\mathbb{R}^{\Lambda}$,

$$
\int g\left(z_{\Lambda}\right) \nu^{t}\left(d z_{\Lambda} \mid y_{\Lambda^{c}}\right)=\int g\left(z_{\Lambda}\right) \mathbf{Q}^{\nu, y_{\Lambda^{c}}}\left(d x, d z_{\Lambda}\right)
$$

From Lemma 10 and Lemma 11, we obtain the existence of a regular density $f_{\Lambda}$ for the conditional probabilities $\nu^{t}\left(\cdot \mid y_{\Lambda^{c}}\right)$ :

$$
\nu^{t}\left(d z_{\Lambda} \mid y_{\Lambda^{c}}\right)=f_{\Lambda}\left(z_{\Lambda} y_{\Lambda^{c}}\right) m^{\otimes \Lambda}\left(d z_{\Lambda}\right)
$$

with

$$
f_{\Lambda}(y)=\int_{\mathbb{R}^{\mathbb{Z}^{d}}} \frac{1}{\mathbf{Z}_{\Lambda}\left(y_{\Lambda^{c}}\right)} \prod_{i \in \Lambda} p_{t}\left(x_{i}, y_{i}\right) \exp \left(-\sum_{\substack{A \subset \mathbb{Z}^{d} \\ A \cap \Lambda \neq \emptyset}} \boldsymbol{\Phi}_{A}^{\beta}(x, y)\right) \bar{Q}^{\nu, y_{\Lambda^{c}}}(d x)
$$

Let us remark that $f_{\Lambda}$ is well defined on the full space $\mathbb{R}^{\mathbb{Z}^{d}}$.

Step 3: Use of Kozlov representation theorem

To complete the proof of Theorem 2, it is enough to show that the local densities $f_{\Lambda}$ - expressed in (49) - of the family of conditional probabilities $\nu^{t}\left(\cdot \mid \mathcal{F}_{\Lambda^{c}}\right)$ are built on an absolutely summable interaction potential. Unfortunately, in this context, we cannot write explicitely the interaction as we did in the section 3 . We will only prove its existence and regularity, using the pioneering work of Kozlov. In [17], Theorem 1, he proved the existence of an absolutely summable interaction under the assumption that for any $\Lambda \subset \mathbb{Z}^{d}, f_{\Lambda}$ satisfies the following properties :

$$
\begin{array}{ll}
\text { (boundedness) } & \exists C_{1}, C_{2}>0 \text { such that } C_{1} \leq \inf _{y \in \mathbb{R}^{\mathbb{Z}^{d}}} f_{\Lambda}(y) \leq \sup _{y \in \mathbb{R}^{\mathbb{Z}^{d}}} f_{\Lambda}(y) \leq C_{2}, \\
\text { (quasilocality) } & \lim _{\Delta \nearrow \mathbb{Z}^{d}} \sup _{\substack{y, \tilde{y} \in \mathbb{R}^{\mathbb{Z}^{d}} \\
y \Delta=\tilde{y} \Delta}}\left|f_{\Lambda}(y)-f_{\Lambda}(\tilde{y})\right|=0 .
\end{array}
$$


The first condition is equivalent to the uniform boundedness of $\ln \left(f_{\Lambda}\right)$ and the second one is the uniform quasilocality.

Proof of the boundedness of $f_{\Lambda}$ : Since, from Lemma $10, \bar{Q}^{\nu, y_{\Lambda^{c}}}$ is a Gibbs measure with associated interaction $\bar{\Phi}^{y_{\Lambda^{c}}}$, one can desintegrate it on $\mathcal{F}_{\Lambda}$ and obtains

$$
f_{\Lambda}(y)=\int \bar{f}_{\Lambda}\left(x_{\Lambda^{c}}, y\right) \bar{Q}^{\nu, y_{\Lambda^{c}}}(d x)
$$

where

$\bar{f}_{\Lambda}\left(x_{\Lambda^{c}}, y\right)=\int_{\mathbb{R}^{\Lambda}} \frac{1}{\mathbf{Z}_{\Lambda}\left(y_{\Lambda^{c}}\right) \tilde{z}_{\Lambda}\left(x_{\Lambda^{c}}\right)} \prod_{i \in \Lambda} p_{t}\left(x_{i}, y_{i}\right) \exp \left(-\tilde{h}_{\Lambda}(x)-\sum_{\substack{A \subset \mathbb{Z}^{d} \\ A \cap \Lambda \neq \emptyset}} \boldsymbol{\Phi}_{A}^{\beta}(x, y)\right) m^{\otimes \Lambda}\left(d x_{\Lambda}\right)$,

with

$$
\begin{aligned}
\tilde{z}_{\Lambda}\left(x_{\Lambda^{c}}\right) & =\int \exp \left(-\tilde{h}_{\Lambda}(x)\right) m^{\otimes \Lambda}\left(d x_{\Lambda}\right), \\
\text { and } \mathbf{Z}_{\Lambda}\left(y_{\Lambda^{c}}\right) & =\int \prod_{i \in \Lambda} p_{t}\left(x_{i}, y_{i}\right) \exp \left(-\sum_{\substack{A \subset \mathbb{Z}^{d} \\
A \cap \Lambda \neq \emptyset}} \boldsymbol{\Phi}_{A}^{\beta}(x, y)\right) m^{\otimes \Lambda}\left(d y_{\Lambda}\right) \bar{Q}^{\nu, y_{\Lambda^{c}}}(d x) .
\end{aligned}
$$

By assumption, the initial Hamiltonian $\tilde{h}$ is uniformly bounded; then there exists $c_{1}>0$ and $c_{2}>0$ such that

$$
\forall x \in \mathbb{R}^{\mathbb{Z}^{d}}, \quad c_{1} \leq \frac{1}{\tilde{z}_{\Lambda}\left(x_{\Lambda^{c}}\right)} \exp \left(-\tilde{h}_{\Lambda}(x)\right) \leq c_{2} .
$$

On the other hand, from Lemma 8 , it is clear that for $\beta$ small enough, there exists $c_{3}>0$ and $c_{4}>0$ such that

$$
\left.\forall x, y \in \mathbb{R}^{\mathbb{Z}^{d}}, \quad c_{3} \leq \exp \left(-\sum_{\substack{A \subset \mathbb{Z}^{d} \\ A \cap \Lambda \neq \emptyset}} \boldsymbol{\Phi}_{A}^{\beta}(x, y)\right)\right) \leq c_{4} .
$$

Then, for any $y_{\Lambda^{c}} \in \mathbb{R}^{\Lambda^{c}}$,

$c_{3} \int \prod_{i \in \Lambda} p_{t}\left(x_{i}, y_{i}\right) m^{\otimes \Lambda}\left(d y_{\Lambda}\right) \bar{Q}^{\nu, y_{\Lambda^{c}}}(d x) \leq \mathbf{Z}_{\Lambda}\left(y_{\Lambda^{c}}\right) \leq c_{4} \int \prod_{i \in \Lambda} p_{t}\left(x_{i}, y_{i}\right) m^{\otimes \Lambda}\left(d y_{\Lambda}\right) \bar{Q}^{\nu, y_{\Lambda^{c}}}(d x)$.

Since, for any $i \in \mathbb{Z}^{d}$ and $x_{i} \in \mathbb{R}, \int p_{t}\left(x_{i}, y_{i}\right) m\left(d y_{i}\right)=1$, we obtain $c_{3} \leq \mathbf{Z}_{\Lambda}\left(y_{\Lambda^{c}}\right) \leq c_{4}$.

This bound implies that, for all $y \in \mathbb{R}^{\mathbb{Z}^{d}}$,

$$
\frac{c_{1} c_{3}}{c_{4}} \leq f_{\Lambda}(y) \leq \frac{c_{2} c_{4}}{c_{3}} .
$$

Proof of the quasilocality of $f_{\Lambda}$ :

Above, we have shown that the function $\bar{f}_{\Lambda}$ defined on $\mathbb{R}^{\Lambda^{c}} \times \mathbb{R}^{\mathbb{Z}^{d}}$ is uniformly bounded. Furthermore, it satisfies

$$
\lim _{\Delta \nearrow \mathbb{Z}^{d}} \sup _{\substack{y, \tilde{y} \in \mathbb{R}^{\mathbb{Z}^{d}} \\ y \Delta=\tilde{y} \Delta}} \sup _{\substack{x \in \mathbb{R}^{\mathbb{Z}^{d}} \\ \text { f }}}\left|\bar{f}_{\Lambda}\left(x_{\Lambda^{c}}, y\right)-\bar{f}_{\Lambda}\left(x_{\Lambda^{c}}, \tilde{y}\right)\right|=0 .
$$

Using the integral representation (50) and (48), one obtains that $f_{\Lambda}$ itself is quasilocal. 
Remark 3 If we remove the ultracontractivity assumption on the self potential $U$, the result of Theorem 2 remains valid, that is for any fixed $t, \nu^{t}$ is strong Gibbsian for $\beta \leq \beta_{0}$, but now the critical value $\beta_{0}$ depends on $t$.

\section{Corollaries and additional remarks}

We proved in the previous sections results on propagation of Gibbsianness. In this last section, we are interested by the propagation of other properties. Does the Strong Dobrushin condition, or the uniqueness of Gibbs measures, or the phase transition property propagate between time 0 and time $t$ ?

We begin with a direct corollary of Theorem 1 .

Corollary 3 Let us consider the system (4) under the assumptions of Theorem 1. If the initial interaction $\tilde{\varphi}$ satisfies $(S D C)$, then for $t$ small enough, the interaction $\varphi^{t}$ at time $t$ satisfies $(S D C)$ too.

\section{Proof :}

The representation (30) shows that $\varphi^{t}$ is a perturbation of $\tilde{\varphi}$. Similarly as in Lemma 8, we obtain that for $t$ small enough $\varphi^{t}$ satisfies (SDC).

In the case of free systems, we can even say something for times $t$ not necessarily small. Let us define the following decoupled dynamics :

$$
\left\{\begin{array}{l}
d X_{i}(t)=d B_{i}(t)-\frac{1}{2} U^{\prime}\left(X_{i}(t)\right) d t, i \in \mathbb{Z}^{d}, t \geq 0 \\
X(0) \simeq \nu
\end{array}\right.
$$

where $\nu$ is a Gibbs measure in $\mathcal{G}(\tilde{\varphi}, m) ; U$ is supposed to be $\mathcal{C}^{2}$ and the measure $e^{-U} d \xi$ can be normalised into $m(d \xi)=\frac{1}{Z} e^{-U(\xi)} d \xi$.

Proposition 4 If we consider the free system (51) where the initial interaction $\tilde{\varphi}$ satisfies $(S D C)$, then for any time $t \geq 0$, the set $\mathcal{G}\left(\varphi^{t}, m\right)$ is reduced to the unique Gibbs measure equal to the law of $X(t)$.

Moreover, if the dynamical self interaction $U$ is ultracontractive, then for $t$ large enough, the interaction at time $t \varphi^{t}$ satisfies Dobrushin uniqueness criterium.

\section{Proof:}

Let $\mu$ be a Gibbs measure in $\mathcal{G}\left(\varphi^{t}, m\right)$. Suppose that $\mu$ is extremal; then, as recalled in Lemma $1, \mu$ is the weak limit of its local specifications, that is : there exists $y$ in $\mathbb{R}^{\mathbb{Z}^{d}}$ such that $\mu=\lim _{\Lambda \nearrow \mathbb{Z}^{d}} f_{\Lambda}\left(\cdot y_{\Lambda^{c}}\right) m^{\otimes \Lambda} \otimes \delta_{y_{\Lambda^{c}}}$, with $f_{\Lambda}$ defined in (49). But the expression (49) is now much simpler than in Section 4 since the system is free $(\beta=0)$. In this special case, the local specification $f_{\Lambda}$ of $\nu^{t}$ in $\Lambda$ has the following simple expression :

$$
f_{\Lambda}(y)=\int \prod_{i \in \Lambda} p_{t}\left(x_{i}, y_{i}\right) \bar{Q}^{\nu, y_{\Lambda^{c}}}(d x) .
$$

So, for all $\Delta \subset \mathbb{Z}^{d}$ and all bounded regular $\mathcal{F}_{\Delta}$-measurable function $g$, we have

$$
\begin{aligned}
\int g\left(z_{\Delta}\right) \mu(d z) & =\lim _{\Lambda \nearrow \mathbb{Z}^{d}} \int g\left(z_{\Delta}\right) \int \prod_{i \in \Lambda} p_{t}\left(x_{i}, z_{i}\right) \bar{Q}^{\nu, y_{\Lambda^{c}}}(d x) m^{\otimes \Lambda}\left(d z_{\Lambda}\right) \\
& =\lim _{\Lambda \nearrow \mathbb{Z}^{d}} \iint g\left(z_{\Delta}\right) \prod_{i \in \Delta} p_{t}\left(x_{i}, z_{i}\right) m^{\otimes \Delta}\left(d z_{\Delta}\right) \bar{Q}^{\nu, y_{\Lambda} c}(d x)
\end{aligned}
$$


since for every $i \in \Lambda \backslash \Delta, \int p_{t}\left(x_{i}, z_{i}\right) m\left(d z_{i}\right)=1$.

On the other hand, Lemma 11, which holds for $\Lambda=\mathbb{Z}^{d}$ too, shows the weak convergence of $\bar{Q}^{\nu, y_{\Lambda^{c}}}$ against $\bar{Q}^{\nu, \emptyset}$, which is equal to $\nu$ when $\beta=0$. Since the function $x \mapsto \int g\left(z_{\Delta}\right) \prod_{i \in \Delta} p_{t}\left(x_{i}, z_{i}\right) m^{\Delta}\left(d y_{\Delta}\right)$ is bounded and local, we then get

$$
\int g\left(z_{\Delta}\right) \mu(d z)=\iint g\left(z_{\Delta}\right) \prod_{i \in \Delta} p_{t}\left(x_{i}, z_{i}\right) m^{\otimes \Delta}\left(d z_{\Delta}\right) \nu(d x) .
$$

The preceeding equality shows that locally, $\mu$ is nothing but the measure $\nu$ transported by the free dynamics. Thus, $\mu$ does not depend on the boundary condition $y$. This proves the uniqueness of the extremal Gibbs measures in $\mathcal{G}\left(\varphi^{t}, m\right)$. Thus the set of Gibbs measures is reduced to one element too. The first part of the proposition is proved.

We now prove the second assertion. For fixed $t>0$, one can define as usually Dobrushin's coefficients $\left(C_{i, j}^{(t)}\right)_{i, j \in \mathbb{Z}^{d}}$ associated to the interaction $\varphi^{t}$ by :

$$
\begin{aligned}
C_{i, j}^{(t)} & =\sup \left\{\left\|\nu^{t}\left(d y_{i} \mid y_{\mathbb{Z}^{d} \backslash i}\right)-\nu^{t}\left(d y_{i} \mid \tilde{y}_{\mathbb{Z}^{d} \backslash i}\right)\right\|_{\mathrm{var}}: y, \tilde{y} \in \mathbb{R}^{\mathbb{Z}^{d}}, y_{\mathbb{Z}^{d} \backslash j}=\tilde{y}_{\mathbb{Z}^{d} \backslash j}\right\} \\
& =\frac{1}{2} \sup \left\{\int\left|f_{i}(y)-f_{i}(\tilde{y})\right| m\left(d y_{i}\right): y, \tilde{y} \in \mathbb{R}^{\mathbb{Z}^{d}}, y_{\mathbb{Z}^{d} \backslash j}=\tilde{y}_{\mathbb{Z}^{d} \backslash j}\right\}
\end{aligned}
$$

with, as in (52), $f_{i}(y)=f_{\{i\}}(y)=\int p_{t}\left(x_{i}, y_{i}\right) \bar{Q}^{\nu, y_{\mathbb{Z}^{d}} \backslash i}(d x)$. To simplify, the Dobrushin's coefficients of $\tilde{\varphi}$ (time 0 ) are denoted by $\left(C_{i, j}\right)_{i, j \in \mathbb{Z}^{d}}$.

The potentiel $\varphi^{t}$ satisfies the Dobrushin Uniqueness Criterium if

$$
C^{(t)}:=\sup _{i \in \mathbb{Z}^{d}} \sum_{j \in \mathbb{Z}^{d}} C_{i, j}^{(t)}<1 .
$$

Since $\tilde{\varphi}$ satisfies (SDC), it's well known (see for example Proposition 8.8 in [13]) that $\tilde{\varphi}$ satisfies (DUC) too, that is $C:=\sup _{i \in \mathbb{Z}^{d}} \sum_{j \in \mathbb{Z}^{d}} C_{i, j}<1$. Let us prove that $\varphi^{t}$ also satisfies (DUC) for $t$ large enough. From Lemma 10, $\bar{Q}^{\nu, y_{\mathbb{Z}^{d} \backslash i}}$ and $\bar{Q}^{\nu, \tilde{y}_{\mathbb{Z}^{d}} \backslash i}$ are Gibbs measures. Using the comparison Theorem 8.20 in [13] which gives bounds for the integral of a function under different Gibbs measures, one obtains

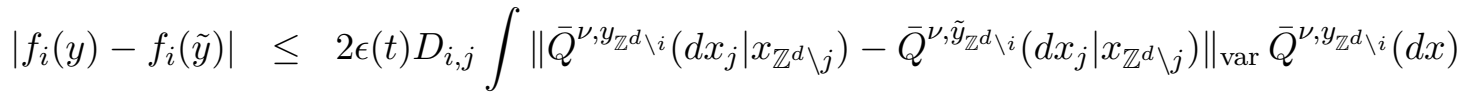

$$
\begin{aligned}
& \leq 2 \epsilon(t) D_{i, j} \frac{1}{2} \iint e^{-\tilde{h}_{j}(x)}\left|\frac{p_{t}\left(x_{j}, y_{j}\right)}{z_{j}\left(x, y_{j}\right)}-\frac{p_{t}\left(x_{j}, \tilde{y}_{j}\right)}{z_{j}\left(x, \tilde{y}_{j}\right)}\right| m\left(d x_{j}\right) \bar{Q}^{\nu, y_{\mathbb{Z}^{d}} \backslash i}(d x),
\end{aligned}
$$

where $\epsilon(t)$ is defined in (38), $D_{i, j}$ is the $(i, j)$-coefficient of the infinite-dimensional matrix $\mathbf{D}=\sum_{n \in \mathbb{N}} \mathbf{C}^{n}\left(\mathbf{C}\right.$ is the matrix $\left.\left(C_{i, j}\right)_{i, j \in \mathbb{Z}^{d}}\right)$ and

$$
z_{j}\left(x, y_{j}\right)=\int e^{-\tilde{h}_{j}(x)} p_{t}\left(x_{j}, y_{j}\right) m\left(d x_{j}\right)
$$

If we denote by $z_{j}(x)=\int e^{-\tilde{h}_{j}(x)} m\left(d x_{j}\right)$ we then obtain following inequalities :

$$
\begin{gathered}
(1-\epsilon(t)) z_{j}(x) \leq z_{j}\left(x, y_{j}\right) \leq(1+\epsilon(t)) z_{j}(x), \\
\left|z_{j}\left(x, y_{j}\right)-z_{j}\left(x, \tilde{y}_{j}\right)\right| \leq 2 \epsilon(t) z_{j}(x) .
\end{gathered}
$$


Thus,

$$
\left|f_{i}(y)-f_{i}(\tilde{y})\right| \leq \epsilon(t) D_{i, j}(A+B)
$$

with

$$
A=\iint e^{-h_{j}(x)} \frac{1}{z_{j}\left(x, y_{j}\right)}\left|p_{t}\left(x_{j}, y_{j}\right)-p_{t}\left(x_{j}, \tilde{y}_{j}\right)\right| m\left(d x_{j}\right) \bar{Q}^{\nu, y_{\mathbb{Z}^{d} \backslash i}}(d x),
$$

and

$$
B=\iint e^{-h_{j}(x)} p_{t}\left(x_{j}, \tilde{y}_{j}\right)\left|\frac{1}{z_{j}\left(x, y_{j}\right)}-\frac{1}{z_{j}\left(x, \tilde{y}_{j}\right)}\right| m\left(d x_{j}\right) \bar{Q}^{\nu, y_{\mathbb{Z} d} \backslash i}(d x) .
$$

Using inequalities (53) and (54) we obtain

$$
\begin{aligned}
A & \leq 2 \epsilon(t) \iint \frac{e^{-h_{j}(x)}}{z_{j}\left(x, y_{j}\right)} m\left(d x_{j}\right) \bar{Q}^{\nu, y_{\mathbb{Z}^{d} \backslash i}}(d x) \\
& \leq \frac{2 \epsilon(t)}{1-\epsilon(t)} \iint \frac{e^{-h_{j}(x)}}{z_{j}(x)} m\left(d x_{j}\right) \bar{Q}^{\nu, y_{\mathbb{Z}^{d}} \backslash i}(d x) \\
& \leq \frac{2 \epsilon(t)}{1-\epsilon(t)}
\end{aligned}
$$

(For $t$ large enough, $1-\epsilon(t)$ is greater than 0 .)

On the other hand,

$$
\begin{aligned}
B & \leq \iint \frac{e^{-h_{j}(x)}}{z_{j}\left(x, \tilde{y}_{j}\right)} p_{t}\left(x_{j}, \tilde{y}_{j}\right) \frac{\left|z_{j}\left(x, \tilde{y}_{j}\right)-z_{j}\left(x, y_{j}\right)\right|}{z_{j}\left(x, y_{j}\right)} m\left(d x_{j}\right) \bar{Q}^{\nu, y_{\mathbb{Z}^{d} \backslash i}}(d x) \\
& \leq \iint \frac{e^{-h_{j}(x)}}{z_{j}\left(x, \tilde{y}_{j}\right)} p_{t}\left(x_{j}, \tilde{y}_{j}\right) \frac{2 \epsilon(t)}{1-\epsilon(t)} m\left(d x_{j}\right) \bar{Q}^{\nu, y_{\mathbb{Z}^{d} \backslash i}(d x)} \\
& \leq \frac{2 \epsilon(t)}{1-\epsilon(t)}
\end{aligned}
$$

Finally, we obtain the uniform bound $\left|f_{i}(y)-f_{i}(\tilde{y})\right| \leq \frac{4 \epsilon(t)^{2}}{1-\epsilon(t)} D_{i, j}$. Thus, for all $i \in \mathbb{N}$,

$$
C^{(t)} \leq \frac{2 \epsilon(t)^{2}}{1-\epsilon(t)} \sum_{j \in \mathbb{N}} D_{i, j} \leq \frac{2 \epsilon(t)^{2}}{1-\epsilon(t)} \frac{1}{1-C}
$$

Since $\epsilon(t)$ vanishes when $t$ goes to infinity, $C^{(t)}$ is strictly lower than 1 for $t$ large enough.

Let us go back to the general system (31), with a true interaction in the dynamics. We know that for small times the set $\mathcal{G}\left(\varphi^{t}, m\right)$ contains a unique Gibbs measure. But it is not clear whether this property remains true for any time. What we prove in the following proposition, is that it is at least true for $t$ large enough. Unfortunately, unlike the preceding Proposition, we do not know if the potentiel $\varphi^{t}$ satisfies the uniqueness criteria (DUC) or (SDC).

Proposition 5 Under the assumptions of Theorem 2, for $\beta$ small enough and $t$ large enough, the set $\mathcal{G}\left(\varphi^{t}, m\right)$ contains a unique Gibbs measure, the law at time $t$ of the solution of (31). 


\section{Proof :}

For $\beta$ small enough, the interaction potential on $\mathbb{R}^{S}$ associated to the Hamiltonian function $\mathbf{H}$ defined in (45) is the sum of the initial potential plus a two-body potential induced by $p_{t}$ and a dynamical potential defined by the cluster expansion. By assumption, the first one satifies (SDC); the second one vanishes when $t$ goes to infinity, since $U$ is ultracontractive and the third one is small in the sense of Lemma 8. So, for $\beta$ small enough and $t$ large enough, the potential associated to $\mathbf{H}$ satisfies (SDC) on the bi-space $S$. Thus, the specifications of $\mathbf{H}$ are global in the sense defined in [11] (see also [12] and [13]). This means that (DLR)-equations hold also true for unbounded subsets of $S$. Similarly to the beginning of the proof of Proposition 4 , we can show that each extremal measure in $\mathcal{G}\left(\varphi^{t}, m\right)$ is the limit of the projections on $\mathbb{R}^{\mathbb{Z}^{d} \times\{1\}}$ of the global specifications associated to $\mathbf{H}$ for a fixed boundary condition $y$. The uniqueness of such extremal Gibbs measures is then a consequence of the globality property. We conclude that $\mathcal{G}\left(\varphi^{t}, m\right)$ is reduced to the measure $\nu^{t}$.

Let us finish this section with a result about propagation of non-uniqueness.

Proposition 6 Let us consider the system (4) under the assumptions of Theorem 1. If $\# \mathcal{G}(\tilde{\varphi}, d x)>1$ (phase transition occurs at time 0 ) then, for t small enough $\# \mathcal{G}\left(\varphi^{t}, d x\right)>1$ too, that is the phase transition propagates.

\section{Proof :}

Suppose $\# \mathcal{G}(\tilde{\varphi}, d x)>1$; let $\nu_{1}$ and $\nu_{2}$ be two distinct measures in $\mathcal{G}(\tilde{\varphi}, d x)$. Thanks to Theorem 1 , for $t$ small enough, $\nu_{1}^{t}$ and $\nu_{2}^{t}$ are in the same set of Gibbs measures $\mathcal{G}\left(\varphi^{t}, d x\right)$. It is clear that $\nu_{1}^{t}$ (respectively $\nu_{2}^{t}$ ) converges weakly to $\nu_{1}$ (respectively to $\nu_{2}$ ) when $t$ goes to 0 . Thus, for $t$ small enough $\nu_{1}^{t}$ and $\nu_{2}^{t}$ are different measures.

Acknowledgements. The authors thank A.C.D. van Enter, A. Le Ny and F. Redig for the very interesting workshop they organized at Eurandom, where the results of this paper were presented for the first time. They also thank R. Fernandez, F. den Hollander and F. Redig for fruitful discussions.

During the completion of this work the first author benefited from the financial support of DAAD (Kurzstipendium A/03/02945) and from the hospitality of Potsdam University. Both institutions are here gratefully acknowledged.

\section{References}

[1] A. Bovier and M. Zahradník, A simple inductive approach to the problem of convergence of cluster expansions of polymer models, J. Statist. Physics, 100 (2000), 765-778.

[2] P. Cattiaux, S. Rolly and H. Zessin, Une approche Gibbsienne des diffusions Browniennes infini-dimensionnelles, Probab. Th. Rel. Fields 104 (1996), 147-179.

[3] P. Dai Pra and S. Roelly, An existence result for infinite-dimensional Brownian diffusions with non-regular and non-Markovian drift, Markov Proc. Rel. Fields 10 (2004), 113-136. 
[4] D. Dereudre and S. Rølly, On Gibbsianness of infinite-dimensional diffusions, Proceedings Conference Eurandom Gibbs versus non-Gibbs, Markov Proc. Rel. Fields 10 (2004), 395-410.

[5] J.D. Deuschel, Non-linear smoothing of infinite-dimensional diffusion processes, Stochastics Vol. 19 (1986), 237-261.

[6] J.D. Deuschel, Infinite-dimensional diffusion processes as Gibbs measures on $C[0,1]^{\mathbb{Z}^{d}}$, Probab. Th. Rel. Fields 76 (1987), 325-340.

[7] H. Doss and G. Royer, Processus de diffusion associé aux mesures de Gibbs, Z. Wahrsch. Verw. Geb. 46 (1978), 125-158.

[8] A.C.D. van Enter, R. Fernandez, F. den Hollander and F. Redig, Possible loss and recovery of Gibbsianness during the stochastic evolution of Gibbs measures, Commun. Math. Phys. 226 (2002), 101-130.

[9] A.C.D. van Enter, R. Fernandez and A.D. Sokal, Regularity properties and pathologies of position-space renormalisation-group transformations : scope and limitations of Gibbsian theory, J. Stat. Phys. 72 (1993), 879-1167.

[10] A.C.D. van Enter and J. Lörinczi, Robustness of non-Gibbsian property : some examples, J. Phys. A 29 (1996), 2465-2473.

[11] R. Fernandez and C.-E. Pfister, Global specifications and nonquasilocality of projections of Gibbs measures, Annals of Prob. 25-3 (1997), 1284-1315.

[12] H. Föllmer, On the global Markov property, In Quantum fields: Algebras, Processes, Ed. Streit, Springer (1980), 293-302.

[13] H.-O. Georgii, Gibbs measures and phase transitions, Berlin, W. de Gruyter (1988).

[14] I.A. Ignatyuk, V.A. Malyshev and V. Sidoravicius, Convergence of the stochastic quantization method $I^{*}$, Theory Prob. Appl. 37-2 (1990), 209-221.

[15] O. Kavian, G. Kerkyacharian and B. Roynette, Quelques remarques sur l'ultracontractivité, J. Func. Anal. 111 (1993), 155-196.

[16] R. Kotecký and D. Preiss, Cluster expansions for abstract polymer models, Commun. Math. Phys. 103 (1986), 491-498.

[17] O.K. Kozlov, Gibbs description of a system of random variables, Probl. Info. Trans. 10 (1974), 258-265.

[18] C. Külske and F. Redig, Loss without recovery of Gibbsianness during diffusion of continuous spins, Preprint 2004

[19] A. Le Ny and F. Redig, Short time conservation of Gibbsianness under local stochastic evolutions, J. Stat. Phys. 109, 5-6 (2002), 1073-1090.

[20] C. Maes and K. Netočný, Space-time expansions for weakly coupled interacting particle systems, J. of Physics A 35 (2002), 3053-3077. 
[21] V.A. Malyshev and R.A. Minlos, Gibbs Random Fields, Cluster expansions, Mathematics and Its Applications Vol. 44, Kluwer Ac. Pub. (1991).

[22] R.A. Minlos, S. Røelly and H. Zessin, Gibbs states on space-time, Potential Analysis 13 (2000), 367-408.

[23] R.A. Minlos, A. Verbeure and V. Zagrebnov, A quantum crystal model in the light mass limit: Gibbs states, Rev. Math. Phys. Vol. 12-7 (2000), 981-1032.

[24] C. Preston, Random fields, L.N. in Math. 534, Springer (1976).

[25] S. Rølly, D. Seu, Limite ergodique de processus de diffusion infini-dimensionnels, Publ. Matematiques 43 (1999), 191-205.

[26] G. Royer, Une initiation aux inegalités de Sobolev logarithmiques, Cours Spécialisés, Soc. Math. France, Paris (1999).

[27] T. Shiga and A. Shimizu, Infinite dimensional stochastic differential equations and their applications, J. Math. Kyoto Univ. 20, 3 (1980), 395-416.

[28] A.L. Toom, N.B. Vasilyev, O.N. Stavskaya, L.G. Mityushin, G.L. Kurdyumov and S.A. Pirogov, Locally interactive systems and their application in biology, L.N. in Math. 653, Springer (1978). 